\title{
A computational approach to identify microRNA (miRNA) based biomarker of Pharmacovariant from the regulation of disease pathology
}

\begin{abstract}
In the post genomic era, identification of a potential miRNA in a computational approach for the significance of a discovery of systemic biomarker with respect to pharmacovarient studies to treat diseases is a challenging task to execute. The challenge was addressed by identifying the Pharmacovarient based associate genes from Verse and it was followed by identifying the associated miRNAs from PharmacomiR and finally the transcription factors(TFs) of associate target genes were identified from RegNetworks. In the next step, a miRNA based regulatory network was constructed on the basis of association between genes, miRNAs and TFs. Finally, the network was analyzed on the basis of statistical studies and miRNA based compatibility to identify a potential miRNA to be utilized as a biomarker of Pharmacovarient to treat diseases in future. In this article, the computational approach was used for the identification of a miRNA based systemic biomarker in association with the pharmacovaience of Psoriasis and in future this approach can also be used for other diseases.
\end{abstract}

Keywords: pharmacovarience; transcription factors; post genomic era; RegNetworks; miRNA; biomarker.
Volume 7 Issue 3 - 2018

\author{
Harishchander Anandaram \\ Department of Bioinformatics, Sathyabama University, India
}

Correspondence: Harishchander Anandaram, Department of Bioinformatics, Sathyabama University, India,

Email harishchander.a@gmail.com

Received: March 22, 2018| Published: May 15, 2018

\section{Introduction}

Psoriasis is a disorder mediated by immune system by making certain faulty signals in the human body. It's still a belief that psoriasis can be developed under the specified condition i.e. "when the immune system signals the body to accelerate the growth of skin cells. In case of psoriasis, the skin cells mature in 3-6days. Instead of being in shed, the cells in skin get pile up to cause the visible lesions. It was also found that the genes that cause psoriasis can determine the reaction of a person's immune system. These genes can either cause psoriasis or other conditions which are immune-mediated like Type-I Diabetes or rheumatoid arthritis. Pathophysiology of psoriasis involves the understanding of the occurrence of prominent pathologies in the major components of skin i.e. the epidermis and the dermis. There are two well established hypotheses about the process that occurs in the development of the disease. The first hypothesis considers psoriasis as a disorder with excessive growth and reproduction of skin cells. Here, the problem is viewed as a fault of the epidermis and its keratinocytes. In second hypothesis, the disease is viewed as an immune-mediated disorder. Here, the excessive reproduction of skin cells is secondary to the factors produced by the immune system. ${ }^{1,2}$

Micro RNA is a family of non coding RNA (ncRNA) which was discovered in 1993, it consist of 19-25 nucleotides and regulates the expression of approximately $30 \%$ of protein-coding miRNAs in humans. ${ }^{3}$ Base pairing at the position of 2-8 nucleotides were relative to the $5^{\prime}$ end of the small RNA to be termed as the "seed" region and it appears to be important for target recognition. ${ }^{3}$ Maturation of miRNAs involves multiple steps and initially two intermediate forms of miRNAs, namely primary (pri-) and precursor (pre-) miRNAs, were produced sequentially. In this process, Drosha (RNase III enzyme) and the double-stranded RNA (dsRNA) binding protein Dgcr8 cleaves the pri-miRNAs to produce a hairpin-shaped pre-
miRNAs that are recognized by Exportin 5 and they are subsequently transported from the nucleus to cytoplasm. There is another RNase III enzyme called Dicer which cleaves the pre-miRNAs to release $\sim 22-\mathrm{nt}$ double-stranded RNA duplexes (namely miRNA/miRNA* duplexes) with 2-nt 3' overhangs. ${ }^{4}$ One strand of a RNA duplex is termed as a mature miRNA which is further loaded into an Argonaute protein in the RNA-induced silencing complex (RISC) to exert its regulatory function on the basis of its binding with the target transcripts. ${ }^{5}$

A unique miRNA can regulate the expression of hundreds of proteins and the expression of a specific protein may be controlled by several miRNAs. ${ }^{6}$ The sequence conservation of most miRNAs lies between the distantly related organisms to suggest the impact of a strong evolutionary pressure ${ }^{7}$ and they have been shown to participate in many fundamental life processes like development, differentiation, organogenesis, growth control and apoptosis. Accordingly, deregulation of miRNA expression has been shown to contribute to cancer, heart diseases, infectious diseases, inflammatory diseases and other medical conditions, making them potential targets for medical diagnosis and therapy. ${ }^{8}$ Initially, Lee had found lin-4 as a regulator of developmental timing in nematode Caenorhabditis elegans. ${ }^{9}$ After several years, Reinhart had discovered lethal-7 (let-7) gene in Caenorhabditis elegans..$^{10}$ At present, 2500 miRNAs are in the human genome. Majority of miRNA are intragenic. ${ }^{11}$ Micro RNAs are initially transcribed as a part of an RNA stem-loop that in turn forms part of a several hundred nucleotides long miRNA precursor miRNA (pri-miRNA). ${ }^{12}$

\section{Results}

Text mining of Genes from Pharmacogenomics and clinical varients were analyzed along with the associated miRNAs from PharmacomiR and transcription factors from Regnetworks and the 
analysis of interaction resulted in the pairing of 101 genes with 576 miRNAs and 638 transcription factors and the results were given in Table 1. A regulatory network was constructed in Cytoscape and the properties of the network were analyzed in the Network Analyzer.
Finally, the hub genes were identified from cytohubba and the miRNA based regulation was analyzed on the basis of seed pairing in miRmap and triplex miRNA along with the experimental evidences from the previous literature.

Table I Psoriasis associated genes, miRNAs and transcription factors with respect to Pharmacovarience from Pharmacogenomics and Clinical varients.

S. No. Genes (Varse) miRNAs (pharmacomiR) Transcription factor (RegNetworks)

\begin{tabular}{|c|c|c|c|}
\hline I & $A B C B I$ & hsa-miR-222, hsa-miR-338-5p \& hsa-miR-45I & $\begin{array}{l}\text { ETSI,HAXI,HIFIA,JUN,NFKBI } \\
\text { RARA,RNF2,SPI,TFAP2A,TP53 } \\
\text { \&WTI }\end{array}$ \\
\hline 2 & CYP3A4 & hsa-miR-22I & SPI \& TFAP2A \\
\hline 3 & CTLA4 & $\begin{array}{l}\text { hsa-miR-200C, hsa-miR-I0I, hsa-miR-I05, hsa-miR-I55, hsa-miR-205, } \\
\text { hsa-miR-380, hsa-miR-384, hsa-miR-429, hsa-miR-432, hsa-miR-449b, } \\
\text { hsa-miR-45I, hsa-miR-496, hsa-miR-5 I6a-3p, hsa-miR-5 I7a, hsa- } \\
\text { miR-65I \& hsa-miR-656 }\end{array}$ & BPTF, STAT5A \& STAT5B \\
\hline 4 & TNF & $\begin{array}{l}\text { hsa-miR-27a, hsa-miR-I30a, hsa-miR-I30b, hsa-miR-I49, hsa-miR-187, } \\
\text { hsa-miR-I 9a, hsa-miR-296-3p, hsa-miR-409-5p, hsa-miR-454 } \\
\text { hsa-miR-5 I6a-5p, hsa-miR-5I 6b, hsa-miR-5 I 9b-3p, hsa-miR-542-3p, } \\
\text { hsa-miR-58I, hsa-miR-592, hsa-miR-599, hsa-miR-654-3p, hsa-miR-770- } \\
\text { 5p, hsa-miR-875-3p, hsa-miR-875-5p \& hsa-miR-939 }\end{array}$ & $\begin{array}{l}\text { AHR, ARNT, ATFI, ATF2, CEBPB, CEBPD, CREBI, } \\
\text { EBFI, EGR I, EGR4, ELKI, ETSI, ETV4, FOS, IKBKB, } \\
\text { IRF5, JUN, NFAT5, NFATCI, NFATC2, NFATC3, } \\
\text { NFATC4, NFE2LI, NFKBI, NFKBI, NFKB2, } \\
\text { POU2FI, RELA, RELA, SMAD6, SMAD7, SPI, } \\
\text { SP3, SPII, STATI, STAT2, STAT3, STAT4, STAT5A, } \\
\text { STAT5B, STAT6, TBP,TFAP2A \& TP53 }\end{array}$ \\
\hline 5 & MTHFR & $\begin{array}{l}\text { hsa-miR-33I-5p, hsa-miR-103, hsa-miR-I07, hsa-miR-I25a-3p, hsa- } \\
\text { miR-I38, hsa-miR-16, hsa-miR-195, hsa-miR-2 I4, hsa-miR-22, hsa-miR- } \\
\text { 220b, hsa-miR-24, hsa-miR-424, hsa-miR-486-3p, hsa-miR-497, hsa-miR- } \\
\text { 516a-5p, hsa-miR-565, hsa-miR-642 \& hsa-miR-646 }\end{array}$ & $\begin{array}{l}\text { AHR, ARNT, CUXI, E2FI, EP300, JUN, MAX, } \\
\text { MXII::CLEC5A, MYC, REST, SPI, SREBFI, SREBF2, } \\
\text { TFAP2A, USFI, XBPI } \\
\text { \&YYI }\end{array}$ \\
\hline & & hsa-miR-45I, hsa-miR-I24 & $\begin{array}{l}\text { BACH2, CREBBP, CTCF, FOS, FOSB, FOSLI, } \\
\text { GTF2B, HMGN3, HNF4A, HR, JUN, JUNB, JUND, }\end{array}$ \\
\hline 6 & VDR & $\begin{array}{l}\text { hsa-miR-I 25b, hsa-miR-506 } \\
\text { \& hsa-miR-544 }\end{array}$ & $\begin{array}{l}\text { KDM5A, LMO2, MAX, MEDI, NCOAI, NCOA2, } \\
\text { NCOA6, } \\
\text { NROB2, NRIH2, NRIPI, RXRA, RXRB, RXRG, } \\
\text { SMAD3, SNWI, STATI \& TRIM24 }\end{array}$ \\
\hline 7 & CREBBP & hsa-miR-27b & $\begin{array}{l}\text { AIRE, ALXI, AR, ATFI, ATF4, BCL3, BRCAI, } \\
\text { CALCOCOI, CDX2, CEBPA, CEBPB, CEBPD, } \\
\text { CITEDI, CITED2, CNOT3, CREBI, CREB3, } \\
\text { CREB3LI, CREB3L3, CREB3L4 \& CREB5 }\end{array}$ \\
\hline 8 & $A B C C 2$ & hsa-miR-I55, hsa-miR-339-5p & CEBPB, HNF4A,PPARG::RXRA, RXRA \& TP53 \\
\hline 9 & UGTIA8 & hsa-miR-I8Ia & MYB \& SRF \\
\hline 10 & UGTIA9 & hsa-miR-I8Ic & CUXI, MYB \& SRF \\
\hline 11 & NRIII & hsa-miR-| I I d, hsa-miR-|48a \& hsa-miR-I48a & $\begin{array}{l}\text { NCOAI, NCOA3, NCOR2, POUIFI, PPARGCIA, } \\
\text { RXRA, RXRB \& RXRG }\end{array}$ \\
\hline 12 & AKTI & $\begin{array}{l}\text { hsa-miR-I8Ib, hsa-miR-I25b, hsa-miR-I49*, hsa-miR-I85, hsa-miR-409- } \\
\text { 3p, hsa-miR-45 I\& hsa-miR-625 }\end{array}$ & $\begin{array}{l}\text { AR, BRCAI, CREBI, EGRI } \\
\text { EP300, ESRI, ESR2, EZH2 } \\
\text { FOXOI, FOXO3, FOXO4 } \\
\text { GATAI, GATA2, HIST2, H2BE, HTT, IKBKB, } \\
\text { IKBKG, MDM4 } \\
\text { NFKBI, NFKBIA, NFKBIB } \\
\text { NKX3-I, NR4AI, PATZI } \\
\text { PHB2, PPARA, PPARG, PPARGCIB, RELA, SMAD2 } \\
\text { SMAD3, SMAD4, SMAD7 } \\
\text { SPI, STATI, STAT5A, TP53, TRIB3, XBPI,YBXI \& } \\
\text { YYI }\end{array}$ \\
\hline
\end{tabular}

AR,CEBPA,CEBPB,EGRI,EGR2,

ETSI,HAXI,HIFIA,JUN,NFKBI RARA,RNF2,SPI,TFAP2A,TP53

sa-miR-22

hsa-miR-45I, hsa-miR-496, hsa-miR-5 I6a-3p, hsa-miR-517a, hsa-

hsa-miR-27a, hsa-miR-130a, hsa-miR-130b, hsa-miR-149, hsa-miR-187, a-miR-19a, hsa-miR-296-3p, hsa-miR-409-5p, hsa-miR-454

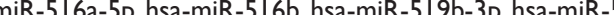
hsa-miR-58I, hsa-miR-592, hsa-miR-599, hsa-miR-654-3p, hsa-miR-770-

MXII::CLEC5A, MYC, REST, SPI, SREBFI, SREBF2, TFAP2A, USFI, XBPI

BACH2, CREBBP, CTCF, FOS, FOSB, FOSLI, GTF2B, HMGN3, HNF4A, HR, JUN, JUNB, JUND, NCOA6,

NROB2, NRIH2, NRIPI, RXRA, RXRB, RXRG,

AIRE,ALXI,AR, ATFI,ATF4, BCL3, BRCAI, CALCOCOI, CDX2, CEBPA, CEBPB, CEBPD, CITEDI, CITED2, CNOT3, CREBI, CREB3, CREB3LI, CREB3L3, CREB3L4 \& CREB5

CEBPB, HNF4A,PPARG::RXRA, RXRA \& TP53

MYB \& SRF

NCOAI, NCOA3, NCOR2, POUIFI, PPARGCIA AR, BRCAI, CREBI, EGR

, ESRI, ESR2, EZH

GATAI, GATA2, HIST2, H2BE, HTT, IKBKB,

IKBKG, MDM4

PHB2, PPARA, PPARG, PPARGCIB, RELA, SMAD2

YY 
Table Continued

S. No. Genes (Varse) miRNAs (pharmacomiR)

hsa-miR-I9a, hsa-miR-103, hsa-miR-107, hsa-miR-I27I, hsa-miR-I30a,

hsa-miR-I30b, hsa-miR-I44, hsa-miR-I5a, hsa-miR-I5b, hsa-miR-I6,

hsa-miR-I8Ia, hsa-miR-I8Ic, hsa-miR-I95, hsa-miR-30 Ia

I3 MAP2K

I4 PIK3CA
hsa-miR-335, hsa-miR-34a,

hsa-miR-34c, hsa-miR-34c-5p,

hsa-miR-4I I, hsa-miR-424, hsa-miR-449a, hsa-miR-497, hsa-miR-50 I5p, hsa-miR-506, hsa-miR-5 I 6a-3p, hsa-miR-545, hsa-miR-575, hsamiR-620, hsa-miR-889 \& hsa-miR-96

hsa-miR-I9b, hsa-miR-I0a, hsa-miR-I0b, hsa-miR-I24, hsa-miR-I35a, hsa-miR-| 35b, hsa-miR-|48a, hsa-miR-|48b, hsa-miR-I52, hsa-miR-I55, hsa-miR-193a-5p, hsa-miR-19a, hsa-miR-19b, hsa-miR-203, hsa-miR302a, hsa-miR-302b, hsa-miR-302c, hsa-miR-302d, hsa-miR-370, hsamiR-372, hsa-miR-373, hsa-miR-422a, hsa-miR-490-5p, hsa-miR-506, hsa-miR-5 I 2-3p, hsa-miR-520a-3p, hsa-miR-520a-5p, hsa-miR-520b, hsa-miR-520d-3p, hsa-miR-520e, hsa-miR-525-5p, hsa-miR-56I, hsamiR-576-5p

\& hsa-miR-942

hsa-miR-30 I b, hsa-let-7a, hsa-miR-I, hsa-miR-I03, hsa-miR-I06a hsa-miR-I07, hsa-miR-I25a-5p, hsa-miR-I25b, hsa-miR- I39-5p, hsamiR-I40-3p, hsa-miR-I43, hsa-miR-I53, hsa-miR-I5a, hsa-miR-I5b, hsa-miR-I6, hsa-miR-I7, hsa-miR-I8Ia, hsa-miR-I8Ib, hsa-miR-I8Ic hsa-miR-I8Id, hsa-miR-I82, hsa-miR-192, hsa-miR-195, hsa-miR-204, hsa-miR-20a, hsa-miR-2I, hsa-miR-2II, hsa-miR-2I7, hsa-miR-23a, hsa-miR-23b, hsa-miR-296-5p, hsa-miR-29a, hsa-miR-29b

hsa-miR-29c, hsa-miR-30a, hsa-miR-30b, hsa-miR-30c, hsa-miR338-5p, hsa-miR-34a, hsa-miR-34b, hsa-miR-34b*, hsa-miR-34c, hsamiR-34c-5p, hsa-miR-365, hsa-miR-424, hsa-miR-429, hsa-miR-448, hsa-miR-45I, hsa-miR-495, hsa-miR-497, hsa-miR-503, hsa-miR-504, hsa-miR-5 I 9a, hsa-miR-5 I 9b-3p, hsa-miR-5 I 9c-3p, hsa-miR-548b-5p, hsa-miR-548c-3p, hsa-miR-548c-5p, hsa-miR-548d-5p, hsa-miR-587, hsa-miR-630, hsa-miR-64I, hsa-miR-646 \& hsa-miR-96

hsa-miR-454, hsa-miR-I43,hsa-miR-I83, hsa-miR-I86, hsa-miR-I94, hsa-miR-205, hsa-miR-25

hsa-miR-298, hsa-miR-378, hsa-miR-422a, hsa-miR-50 I-3p, hsa-miR502-3p, hsa-miR-543, hsa-miR-563, hsa-miR-577, hsa-miR-874

\& hsa-miR-9

hsa-miR-30la, hsa-miR-I36, hsa-miR-28-5p, hsa-miR-33b \& hsamiR-619

hsa-miR-I30a, hsa-miR-I8Ic

hsa-miR-|8Id, hsa-miR-22।

hsa-miR-222 \& hsa-miR-765

hsa-miR-| 30b, hsa-miR-194, hsa-miR-205, hsa-miR-2I0, hsa-miR-224, hsa-miR-300, hsa-miR-30a, hsa-miR-30d, hsa-miR-30e, hsa-miR-335, hsa-miR-452, hsa-miR-494, hsa-miR-502-5p, hsa-miR-543, hsa-miR-570 \& hsa-miR-802

hsa-miR-24, hsa-miR-I 35a, hsa-miR-I 35b, hsa-miR-I 36, hsa-miR-I 50, hsa-miR-I85, hsa-miR-205, hsa-miR-326, hsa-miR-330-5p, hsa-miR-34b, hsa-miR-34c-3p hsa-miR-36I-3p, hsa-miR-495

hsa-miR-498, hsa-miR-539, hsa-miR-542-5p, hsa-miR-626, hsa-miR-660 \& hsa-miR-873

Transcription factor (RegNetworks)

ATFI,ATF2, ATF3, ATF4, ATF5

ATF6, ATF7, CREBI, ELKI, EP300, MYC, POU3F2 \& SPI

CTCF, ESRI, FOXII, FOXOI, FOXO3, FOXO3B FOXO4, PGR

\& TP53

AR, ATFI, BCLAFI, BRCAI, CEBPA, CREBI, CTCF, CUXI, DDIT3, EGRI, ETSI, GLII, GLI2

MYB, MYBLI, MYC, NFKBI, NFKB2, NR4AI, PARPI, PML

PPARG, RARA, RARB, RARG

RELA, SFI, SPI, STAT3, STAT5A

TP53 \&WTI

CEBPB, FOXO3, FOXO3B \& HNFIA

JUN

CREBI, ETSI, ETS2 \& MYB

CUXI, FOXO3, FOXO3B, FOXO4

GTF3CI, NKX6-I, POU3FI, POU3F2, SREBFI, SREBF2

SRY \& TCF3

AR, ARNT, BRCAI, BRCAI, CEBPA, CEBPB CREBBP, CUXI, EGRI, EP300, FOS, IDI, ID2, ID3, KLF4, MAX, MXII::CLEC5A, MYB, MYC, NFKBI, NFYA, PIAS2, SPI, SRF, STATI, STAT2, TFAP2A, TFAP2C \& USFI 
Table Continued

S. No. Genes (Varse)

miRNAs (pharmacomiR)

hsa-miR-I 5b, hsa-miR-|25a-3p, hsa-miR-|27|, hsa-miR-|83, hsa-

hsa-miR-376b, hsa-miR-380, hsa-miR-452, hsa-miR-486-3p, hsa-

miR-489, hsa-miR-569, hsa-miR-583

hsa-miR-586, hsa-miR-604, hsa-miR-624, hsa-miR-639, hsa-miR-646,

hsa-miR-889, hsa-miR-92।

hsa-miR-939, hsa-miR-944\& hsa-miR-96

hsa-miR-497, hsa-miR-2 I 6b, hsa-miR-296-3p, hsa-miR-376c, hsa-miR574-3p, hsa-miR-602, hsa-miR-604, hsa-miR-608, hsa-miR-63 I

\& hsa-miR-94

23

LCK

hsa-miR-424 \& hsa-miR-202

hsa-miR-| 6, hsa-miR-I24, hsa-miR-I28, hsa-miR-I28a, hsa-miR-I28b hsa-miR-I36, hsa-miR-I37, hsa-miR-I4I, hsa-miR-I5b, hsa-miR-I6 hsa-miR-195, hsa-miR-200a, hsa-miR-2 I8, hsa-miR-22, hsa-miR-24 hsa-miR-25, hsa-miR-27a, hsa-miR-27b, hsa-miR-29a, hsa-miR-29b, hsamiR-29c, hsa-miR-30b, hsa-miR-30d, hsa-miR-30e, hsa-miR-32 hsa-miR-363, hsa-miR-367, has-miR-374a, hsa-miR-424, hsa-miR-487b, hsa-miR-497, hsa-miR-503, hsa-miR-505, hsa-miR-506, hsa-miR-580, hsa-miR-892a, hsa-miR-92, hsa-miR-92a, hsa-miR-92b \& hsa-miR-938

hsa-miR-195, hsa-let-7a, hsa-let-7b, hsa-let-7c, hsa-let-7d, hsa-let-7e hsa-let-7f, hsa-let-7g, hsa-let-7i,

hsa-miR-I, hsa-miR-I06a, hsa-miR-I06b, hsa-miR-I24, hsa-miR-I29-3p

hsa-miR-I 29-5p, hsa-miR-I36,

hsa-miR-I37, hsa-miR-I7, hsa-miR-I8a, hsa-miR-I8b, hsa-miR-190, hsamiR-192, hsa-miR-193a-3p, hsa-miR-193b, hsa-miR-I9a, hsa-miR-I9b, hsa-miR-200b, hsa-miR-200c, hsa-miR-203, hsa-miR-206, hsa-miR-20a, hsa-miR-20b, hsa-miR-2I, hsa-miR-23a, hsa-miR-23b hsa-miR-30a, hsa-miR-3I, hsa-miR-340, hsa-miR-37I-5p, hsa-miR-372 hsa-miR-4I I, hsa-miR-429, hsa-miR-433, hsa-miR-488, hsa-miR-495, hsa-miR-499-3p, hsa-miR-506 hsa-miR-5 I7b, hsa-miR-519d, hsa-miR-520d-5p, hsa-miR-524-5p, hsa-miR-544, hsa-miR-548c-3p, hsa-miR-557, hsa-miR-582-5p, hsa-miR-590-5p, hsa-miR-607, hsamiR-6I3, hsa-miR-643, hsa-miR-892b, hsa-miR-9, hsa-miR-93 \& hsamiR-98

hsa-miR-2 | 4, hsa-miR-I0I, hsa-miR-I 22, hsa-miR-I37, hsa-miR-| 42-3p, hsa-miR-|44, hsa-miR-I55, hsa-miR-|82, hsa-miR-I94 hsa-miR-200c, hsa-miR-2I7 hsa-miR-299-5p, hsa-miR-300 hsa-miR-320, hsa-miR-36I-5p hsa-miR-369-3p, hsa-miR-374a hsa-miR-429, hsa-miR-485-3p hsa-miR-5 I8f, hsa-miR-655 \& hsa-miR-96
Transcription factor (RegNetworks)

ALXI, BCL3, CREBI, CTNNBI

EGRI, HLF, HNRNPK, KHDRBSI NFIL3, NFKBI, NKX2-2, NR3C

PAX5, RFXI, RREBI, SPI, STATI

VSX2 \&YTHDCI

CTCF, PAX2, PPARG, TFAP4 \& YYI

ARNT, CTCF, EBFI, ELK I

ESRI, ESR2, ETSI, HNF4A

KHDRBSI, MIF, MYB, NFKBIA

NR3CI, RXRA, SPI, STATI, STAT3, STAT5A, TALI, TCF3 \& USFI

\section{AHR, ARID5B, ARNT, PAX5 \&}

YYI

CEBPB, EGRI, IKBKB, IKBKG NFKBI, RELA \& SPII

E2FI, EGRI, GATAI \& MXII::CLEC5A

ABLI, E2FI, EIF2AK2, ELKI, GABPA, NFKBI,

RELA, STATI

STAT3 \&YYI 
Table Continued

S. No. Genes (Varse) miRNAs (pharmacomiR)

hsa-miR-22, hsa-miR-125b, hsa-miR-135a, hsa-miR- I 35b, hsa-miR-I 5 a

28

RAFI

29

RELA

30

CLAR

CAMK4

32

CAIKKI

33

EDNI

34

GATA4

hsa-miR-302c, hsa-miR-I, hsa-miR-200b, hsa-miR-200c, hsa-miR-208b \& hsa-miR-429

hsa-miR-544, hsa-miR-124, hsa-miR-I 38, hsa-miR-22, hsa-miR-302a, hsa-miR-302b, hsa-miR-302c

hsa-miR-302d, hsa-miR-329, hsa-miR-362-3p, hsa-miR-37I-5p, hsamiR-373, hsa-miR-506, hsa-miR-5 I 2-3p, hsa-miR-520f, hsa-miR-569 hsa-miR-596, hsa-miR-7, hsa-miR-769-5p \& hsa-miR-89Ib miR-5 I 6a-5p, hsa-miR-627, hsa-miR-660 \& hsa-miR-768-5p

hsa-miR-302d, hsa-miR-I06a, hsa-miR-I29-5p, hsa-miR-I7, hsamiR-I87, hsa-miR-191, hsa-miR-20a

hsa-miR-299-3p, hsa-miR-30a

hsa-miR-30b, hsa-miR-30c, hsa-miR-33a \& hsa-miR-33b, hsa-miR-34b, hsa-miR-34c-3p, hsa-miR-455-5p, hsa-miR-507, hsa-miR-5I I, hsamiR-567, hsa-miR-586, hsa-miR-885-5p \& hsa-miR-96 hsa-miR-222, hsa-miR-36 I-3p, hsa-miR-5 I 5-5p, hsa-miR-574-5p, hsamiR-596, hsa-miR-6I9, hsa-miR-63I, hsa-miR-634, hsa-miR-67I-5p, hsa-miR-769-5p, hsa-miR-92I \& hsa-miR-943

hsa-miR-302a, hsa-let-7b, hsa-let-7c, hsa-miR-I, hsa-miR-I47, hsamiR-I 55, hsa-miR-I85, hsa-miR-I 99a-5p, hsa-miR-203, hsa-miR-206, hsa-miR-220c, hsa-miR-454, hsa-miR-5 I 7b, hsa-miR-574-5p, hsamiR-6I3

hsa-miR-648, hsa-miR-892a

\& hsa-miR-935
Transcription factor (RegNetworks)

hsa-miR-302e, hsa-miR-324-3p, hsa-miR-36I-3p, hsa-miR-450b-3p, hsa-

hsa-miR-302b, hsa-miR-I03, hsa-miR-I07, hsa-miR-I45, hsa-miR-22I,

hsa-miR-I8Id, hsa-miR-199a-5p, hsa-miR-199b-5p, hsa-miR-224, hsamiR-26a, hsa-miR-26b, hsa-miR-548a-5p, hsa-miR-548b-5p, hsa-miRFOSB, HAND2, JARID2, JUN, JUNB 548c-5p, hsa-miR-548d-5p, hsa-miR-582-3p, hsa-miR-647 \& hsa-miR876-3p

\section{AR, MYC, NR3CI, RBI, RBL2, RFXANK, TSC22D3} $\& Y Y I$

AATF, AES, AHR, AR, BRCAI, CEBPB, CEBPD, CREBBP, EGRI, EGRI, EP300, ESRI, FOS, FUS, GTF2B, HDACI, HDAC2, HDAC3, HEXIMI, HMGA2, IKBKB, IKBKG, ING4, IRFI, IRF2, IRF3, JUN, KAT2B, MENI, MYC, NCOA3, NCOR2, NFKBI, NFKB2, NFKB2, NFKBIA, NFKBIB, NKRF, NKX2-I, NR3CI, PARPI, PAX6, PDCDII, PGR, PIASI, PIAS3, PML, POU2FI, PPARA, PPARD, REL, RELB, RFCI, RXRA, SIN3A, SIRTI, SNIPI, SPI, SRF, STATI, STAT3, STAT6, TAFI, TAFI I, TAF4B, TAF6, TBP,TP53, TP53BPI, TRIB3, TRIP4, TSC22D3 \& TWISTI

AR, E2FI, E2F4, GABPA, MYC NFYA, NKX2-I, NR3CI, RREBI SPI, TFAP2A, TFAP2C, USFI VSX2 \&YYI

ATF4, BACHI, BACH2, CREBBP EP300, HDAC4, MAX, MEF2A MYC, PPARG \& RELA

ARNT, BACHI, BACH2, E2FI, MAX, MXII::CLEC5A, MYC, NFYA, PAX6, PPARG, TFAP2A

TFAP2C \& USFI

AR, CEBPA, CREBI, E2FI, FOXJ2

FOXOI, HIFIA, HLF, JUN, MEF2A, NFIC, NFKBI, PPARA, PPARG, SMADI, SMAD3, SMAD4, TCF3 \& ZEBI

ATFI,ATF2, ATF3, ATF4, ATF5 ATF6,ATF7, CEBPA, CREBI, CUXI, EP300, FOS, JUND, MEDI, MEF2C, NFATC4 NFKBI, NFKB2, NKX2-5 NR5AI, PIASI, RELA, SRF,TBX5 USFI\& ZFPM2

APC, AR, ARNT, ATF4, BCL3, CDX2, CEBPA, CEBPA, CREBI,

CREB3, CREB3LI, CREB3L3,

CREB3L4, CREB5, CREM, CSDA, CTNNBI, CUXI, E2FI, E2FI, FOXOI, GLII, GLI2, GLI3, HNRNPD, HOXA9, IKBKG, MEISI, MITF, MYC, MYOCD, NFAT5, NFATCI

NFATC2, NFATC3, NFATC4 NFKBI, NFYA, NFYB, NFYC NKX2-2, NOTCH2, PAX5, POU2FI, PPARGCIA, SNAII

SOX9, SPII, STAT5A,TP53 \& YBXI 
Table Continued

\section{S. No. Genes (Varse) \\ miRNAs (pharmacomiR) \\ Transcription factor (RegNetworks)}

hsa-miR-520a-3p, hsa-let-7a, hsa-let-7b, hsa-let-7c, hsa-let-7d, hsa-let7e, hsa-let-7f, hsa-let-7g, hsa-let-7i hsa-miR-196a, hsa-miR-1 96b, hsa-miR-25, hsa-miR-32, hsa-miR-335, hsa-miR-363, hsa-miR-367, hsa-miR-92, hsa-miR-92a, hsa-miR-98

hsa-miR-520e, hsa-miR-I, hsa-miR-I28, hsa-miR-I35b, hsa-miR-I405p, hsa-miR-I8Ia, hsa-miR-I8Ib, hsa-miR-I8Ic, hsa-miR-I8Id, hsamiR-202, hsa-miR-206, hsa-miR-25

hsa-miR-296-5p, hsa-miR-32, hsa-miR-363, hsa-miR-367, hsa-miR-5 I 9a, hsa-miR-5 I 9b-3p, hsa-miR-5 I 9c-3p, hsa-miR-543, hsa-miR-566 hsa-miR-6 I5-5p, hsa-miR-66I, hsa-miR-767-3p, hsa-miR-876-3p, hsamiR-92, hsa-miR-92a \& hsa-miR-92b

hsa-miR-373, hsa-miR-I 24, hsa-miR-I 28, hsa-miR-I 34, hsa-miR-30a, hsa-miR-30b, hsa-miR-506, hsa-miR-589, hsa-miR-629, hsa-miR-653, hsa-miR-765, hsa-miR-768-3p \& hsa-miR-9

hsa-miR-520c-3p, hsa-miR-I24, hsa-miR-|47, hsa-miR-I82, hsa-miRI90b, hsa-miR-199b-5p, hsa-miR-19a, hsa-miR-26a, hsa-miR-26b, hsamiR-27a, hsa-miR-27b, hsa-miR-299-5p, hsa-miR-302b, hsa-miR-302c, hsa-miR-30a, hsa-miR-30a-5p, hsa-miR-30b, hsa-miR-30c, hsa-miR-30d, hsa-miR-30e, hsa-miR-32, hsa-miR-338-5p, hsa-miR-365, hsa-miR-377, hsa-miR-383, hsa-miR-409-5p,hsa-miR-452,hsa-miR-506,hsa-miR-5 I4, hsa-miR-5 I 5-5p, hsa-miR-542-5p, hsa-miR-558, hsa-miR-768-3p, hsamiR-89la \& hsa-miR-96

hsa-miR-520b, hsa-miR-I25a-5p, hsa-miR-I25b, hsa-miR-I297, hsamiR-I8Ib, hsa-miR-I8Id, hsa-miR-I99a, hsa-miR-199a-5p, hsa-miR-26a, hsa-miR-28-5p, hsa-miR-29a, hsa-miR-29b, hsa-miR-29c, hsa-miR-328, hsa-miR-33I-3p, hsa-miR-346, hsa-miR-494, hsa-miR-504, hsa-miR-6545p, hsa-miR-874 \& hsa-miR-93

hsa-miR-520d-3p, hsa-miR-I 35a, hsa-miR-I35b, hsa-miR-I37, hsamiR-I53, hsa-miR-182, hsa-miR-183, hsa-miR-18a, hsa-miR-18b, hsa-miR-190, hsa-miR-190b, hsa-miR-194, hsa-miR-19a, hsa-miR-19b, hsa-miR-203, hsa-miR-2I, hsa-miR-223, hsa-miR-23a, hsa-miR-23b, hsa-miR-27b, hsa-miR-302a, hsa-miR-302b, hsa-miR-302c, hsa-miR302d, hsa-miR-330-3p, hsa-miR-33 I-5p, hsa-miR-33a, hsa-miR-340, hsa-miR-372, hsa-miR-42 I, hsa-miR-448, hsa-miR-450b-5p, hsa-miR520a-3p, hsa-miR-520b, hsa-miR-520c-3p, hsa-miR-520d-3p, hsa-miR520d-5p, hsa-miR-520e, hsa-miR-520f, hsa-miR-524-5p, hsa-miR-556-3p, hsa-miR-603, hsa-miR-62I \& hsa-miR-656

hsa-miR-I 24, hsa-miR-490-5p hsa-miR-593 \& hsa-miR-876-5p

hsa-miR-506, hsa-let-7a,hsa-let-7b

hsa-let-7c, hsa-let-7d, hsa-let-7e, hsa-let-7f, hsa-let-7g, hsa-let-7i, hsamiR-I22,hsa-miR-I55,hsa-miR-2 I

hsa-miR-220c,hsa-miR-569, hsa-miR-574-5p \& hsa-miR-98

hsa-miR-23a, hsa-miR-I47, hsa-miR-I8Ia, hsa-miR-I8I c, hsa-miR-2 I4, hsa-miR-27a, hsa-miR-27b, hsa-miR-323-5p, hsa-miR-374b, hsamiR-375, hsa-miR-382, hsa-miR-4 10, hsa-miR-486-3p, hsa-miR-499-3p, hsa-miR-504, hsa-miR-885-3p \& hsa-miR-944
FHL2, FOXDI, FOXF2, HEY2, HEYL, HOXA9, MEISI, MYODI, POU3FI, RORA, TCFI 2 \& TCF3

AHR, ARNT, BPTF, CENPB, COPS5, EP300, FOXD3, GATA4

HEY2, HEYL, NPMI, PHOX2A

SRY,TCF3 \& VSX2

ANKRA2, BCL6, BCOR, CIITA, HR, KLF4, MAX, MEF2A, MEF2C MEF2D, NCORI, NCOR2, NFKB2 NRIPI, PHB2, RFXANK, RUNX3 USFI \& ZBTBI6

KLFI 2, MEF2A, MEF2A, NCOR REST, SIN3A, SIN3B, USFI, ZBTBI 6 \& ZEB I

CTCF, EGR3, ETSI, ETS2, MZFI NFKBI, PBXI, RREBI, STATI STAT2 \& TBP

ATF2, BPTF, CDC5L, CREBI, CUXI, EP300, ESR I, FOXD3, FOXII, FOXJ2, GATA4, HDAC4 HDAC5, HLF, IRFI, KLFI2, MEF2A, MEF2D, NCOA2,NKX2-2

NKX3-I,PAX6,PBXI,POU2FI, POU2F2,POU3FI,POU3F2,POU3F3 POU5FI,SMAD2,SPI,SPI,SPII, STAT5A,STAT5B,TCF3,TEAD I TFAP4,TGIFI,TWIST2 \& VGLL2

HNFIA \& MEF2A

ABLI, E2F4, ELKI, HCLSI, MEF2A, NR3C2, REST, SRF,TBP

TP53 \& ZEBI

EGRI, EGR3, GABPA, PPARD

$R X R A, R X R B, R X R G$ \& SPI 
Table Continued

\begin{tabular}{|c|c|c|}
\hline S. No. & Genes (Varse) & miRNAs (pharmacomiR) \\
\hline 45 & RPS6KBI & $\begin{array}{l}\text { hsa-miR-23b, hsa-miR-I03, hsa-miR-I07, hsa-miR-I24, hsa-miR-I28, } \\
\text { hsa-miR-I29-5p, hsa-miR-I30a } \\
\text { hsa-miR-I 30b, hsa-miR-I35a, hsa-miR-I35b, hsa-miR-I37, hsa-miR-I42- } \\
\text { 5p, hsa-miR-I45, hsa-miR-I53 } \\
\text { hsa-miR-I55, hsa-miR-I8Ia, hsa-miR-I8I b, hsa-miR-I8I c, hsa-miR- } \\
\text { I8Id, hsa-miR-I86, hsa-miR-200b } \\
\text { hsa-miR-200c, hsa-miR-2 I8, hsa-miR-223, hsa-miR-27a, hsa-miR-27b, } \\
\text { hsa-miR-30 Ia, hsa-miR-30 Ib } \\
\text { hsa-miR-340, hsa-miR-369-3p, hsa-miR-377, hsa-miR-429, hsa-miR-506, } \\
\text { hsa-miR-507, hsa-miR-548c-3p, hsa-miR-557, hsa-miR-56I, hsa- } \\
\text { miR-568, hsa-miR-579, hsa-miR-590-3p, hsa-miR-605, hsa-miR-607, } \\
\text { hsa-miR-64I, hsa-miR-655, hsa-miR-7 \& hsa-miR-944 }\end{array}$ \\
\hline
\end{tabular}

hsa-miR-I25b, hsa-miR-I03, has-miR-I07, hsa-miR-I37, hsa-miR-I5a, hsa-miR-I 5b, hsa-miR-16, hsa-miR-195, hsa-miR-25, hsa-miR-28-3p, hsa-miR-33, hsa-miR-33a, hsa-miR-33b, hsa-miR-36I-5p, hsa-miR-365, hsa-miR-519d, hsa-miR-52I, hsa-miR-523, hsa-miR-563, hsa-miR-5765p,hsa-miR-583, hsa-miR-584, hsa-miR-586, hsa-miR-598, hsa-miR-652, hsa-miR-660, hsa-miR-708, hsa-miR-885-5p, hsa-miR-888, hsa-miR-95\& hsa-miR-96

hsa-miR-I25a-5p, hsa-miR-I, hsa-miR-I6, hsa-miR-I82, hsa-miR-I9a hsa-miR-19b, hsa-miR-206, hsa-miR-28-3p, hsa-miR-383, hsa-miR-503, hsa-miR-5 I 4, hsa-miR-548a-3p

hsa-miR-548a-5p, hsa-miR-548b-5p

hsa-miR-548c-5p, hsa-miR-548d-5p

hsa-miR-56I, hsa-miR-609, hsa-miR-642, hsa-miR-922, hsa-miR-923 \& hsa-miR-96

hsa-miR-590-3p, hsa-miR-449a, hsa-miR-449b, hsa-let-7a, hsa-let-7e, hsa-let-7f, hsa-miR- I06a, hsa-miR-I 06b, hsa-miR-I 25a-5p, hsa-miRI30a, hsa-miR-I30b, hsa-miR-I32, hsa-miR-|45, hsa-miR-|46a, hsamiR-I 46b-5p, hsa-miR-17, hsa-miR-I7-5p,hsa-miR-I82, hsa-miR-193a5p,hsa-miR-20, hsa-miR-208a

hsa-miR-208b, hsa-miR-20a, hsa-miR-20b, hsa-miR-2।, hsa-miR-2। 2 , hsa-miR-22, hsa-miR-224, hsa-miR-28-5p, hsa-miR-298, hsa-miR-299-5p, hsa-miR-30 la, hsa-miR-30 lb

hsa-miR-302a, hsa-miR-345, hsa-miR-363, hsa-miR-370, hsa-miR-372, hsa-miR-423-3p,

hsa-miR-454, hsa-miR-503, hsa-miR-509-3-5p, hsa-miR-5I 5-3p, hsamiR-5 I 6a-3p, hsa-miR-5 I9a, hsa-miR-5 I9b-3p, hsa-miR-519c-3p, hsa-miR-5 I9d, hsa-miR-5 I9e, hsa-miR-520a-3p, hsa-miR-520b, hsamiR-520g, hsa-miR-520h, hsa-miR-572, hsa-miR-608, hsa-miR-639, hsamiR-64I, hsa-miR-654-3p, hsa-miR-657, hsa-miR-93, hsa-miR-939 hsa-miR-942 \& hsa-miR-96

hsa-miR-38I, hsa-miR-I 42-3p, hsa-miR- I82, hsa-miR-423-5p, hsamiR-49I-3p, hsa-miR-574-3p, hsa-miR-58I, hsa-miR-587, hsa-miR-642, hsa-miR-886-3p \& hsa-miR-96

hsa-miR-300, hsa-miR-I, hsa-miR-I 28a, hsa-miR-I 28b, hsa-miR-I 38 , hsa-miR-I42-3p, hsa-miR-I43, hsa-miR-I44, hsa-miR-I82, hsa-miR200b, hsa-miR-200c, hsa-miR-205,

hsa-miR-206, hsa-miR-2I, hsa-miR-2 I6a, hsa-miR-2I 8, hsa-miR-222, hsa-miR-23a, hsa-miR-25, hsa-miR-27a, hsa-miR-27b, hsa-miR-300,
Transcription factor (RegNetworks) hsa-miR-30a, hsa-miR-30a-5p, hsa-miR-30b, hsa-miR-30c, hsa-miR-30d, hsa-miR-30e, hsa-miR-370, hsa-miR-429, hsa-miR-49I-5p, hsa-miR-495, hsa-miR-499-5p, hsa-miR-56I, hsa-miR-655, hsa-miR-770-5p \& hsamiR-876-3p

ABLI, ATF2, CREB I, CREBBP, E2F4, EGR3, ESR I, ESR2, ING I, JUN, MDM4, MEDI, MEF2A, MYC, NCOAI, NCOA2, NCOA3, NR3CI, NRIPI, TFAP2A, THRA, TLX2,TNFAIP3,YYI \& ZFP36
$\mathrm{E} 2 \mathrm{FI}, \mathrm{E} 2 \mathrm{~F} 2$ \& LMO2

CTCF, EGRI, EGR2, EGR3

KLFI2, SPI,TFAP2A \& TFAP2C

AR, ATF2, CDC5L, CEBPA, CEBPA, ESRI, ESR I, ETSI, FLII, FOS, FOXCI, FOXDI, FOXF2, FOXI2, HDACI I, HIFIA, HNF4A, JUN, PARPI, PPARG, RARA, RBI, RELB, SMADI, SMAD2, SMAD3, SMAD4, SPI, SP3, SP4, SRF, STATI, STAT3, TFAP2A, TP53,TP53,TP63,TP73,TSG I0I,WTI\&XRCC6

CTCF, CUXI, EGRI, MEF2A \& PPARG

AHR, ALXI, ARNT, HNFIA MZFI, NFKBI, PAX6, POU2FI TOBI \&YYI 
Table Continued

\begin{tabular}{|c|c|c|c|}
\hline S. No. & Genes (Varse) & miRNAs (pharmacomiR) & Transcription factor (RegNetworks) \\
\hline 51 & SPI & $\begin{array}{l}\text { hsa-miR-I 53, hsa-miR-I, hsa-miR-I05, hsa-miR-I24, hsa-miR-I28, hsa- } \\
\text { miR-I28a, hsa-miR-I 28b, hsa-miR-I35a, hsa-miR-I35b, hsa-miR-I37, } \\
\text { hsa-miR-I38, hsa-miR-I49, hsa-miR-I50, hsa-miR-I 55, hsa-miR-203, } \\
\text { hsa-miR-204, hsa-miR-206, hsa-miR-2II, hsa-miR-2 I6a, } \\
\text { hsa-miR-2 I8, hsa-miR-22, hsa-miR-223, hsa-miR-24, hsa-miR-27a, } \\
\text { hsa-miR-27b, hsa-miR-29a, hsa-miR-29b, hsa-miR-29c, hsa-miR-300, } \\
\text { hsa-miR-32, hsa-miR-320, } \\
\text { hsa-miR-324-5p, hsa-miR-335, } \\
\text { hsa-miR-36I-5p, hsa-miR-362-3p, } \\
\text { hsa-miR-374b, hsa-miR-375, hsa-miR-377, hsa-miR-38I, hsa-miR-384, } \\
\text { hsa-miR-4I0, hsa-miR-429 } \\
\text { hsa-miR-487a, hsa-miR-488, hsa-miR-495, hsa-miR-506, hsa-miR-539, } \\
\text { hsa-miR-548a-5p, hsa-miR-548b-3p, hsa-miR-548b-5p, hsa-miR-548c-5p, } \\
\text { hsa-miR-548d-5p, hsa-miR-570, hsa-miR-590-3p, } \\
\text { hsa-miR-603, hsa-miR-607, hsa-miR-6I3, hsa-miR-625 \& hsa-miR-7 }\end{array}$ & $\begin{array}{l}\text { AATF, AHR, AR, ARNT, ATF7IP } \\
\text { BCL6, BCOR, BRCAI, CEBPA, CEBPB, E2FI, E2F2, } \\
\text { E2F3, E2F4, EGRI, EGRI, EGR3, ELFI, EP300, ESRI, } \\
\text { ESR2, ETSI, GABPA, GATAI, HCFCI, HDACI, } \\
\text { HDAC2, HIFIA, HMGAI, HNF4A, HTT, JUN, } \\
\text { KLFI0, KLF4, KLF6, MEF2A, MEF2C, MEF2D, } \\
\text { MISI8BPI, MSXI, MYB, MYC, MYODI, MYOG, } \\
\text { NCORI, NCOR2, } \\
\text { NFYA, NFYB, NR2FI, NR5AI, } \\
\text { PAX6, PER3, PML, POU2FI, POU2FI, PURA, } \\
\text { RARA, RBI, RBBP4, RBLI, REL, RELA, } \\
\text { RREBI, RXRA, SMAD2, SMAD3 } \\
\text { SMAD4, SOXI0 \& SOX8 }\end{array}$ \\
\hline 52 & SP3 & $\begin{array}{l}\text { hsa-miR-36 I-5p, hsa-miR-I05, hsa-miR-I 24, hsa-miR-I 33a, hsa-miR- } \\
\text { I33b, hsa-miR-I 35a, hsa-miR-I 35b } \\
\text { hsa-miR-I45, hsa-miR-I53, hsa-miR-I 55, hsa-miR-I 82, hsa-miR-223, } \\
\text { hsa-miR-27a, hsa-miR-338-5p } \\
\text { hsa-miR-369-3p, hsa-miR-374a } \\
\text { hsa-miR-374b, hsa-miR-4 I0, hsa-miR-42 I, hsa-miR-448, hsa-miR-487a, } \\
\text { hsa-miR-487b, hsa-miR-506 } \\
\text { hsa-miR-507, hsa-miR-520d-5p, } \\
\text { hsa-miR-522, hsa-miR-524-5p, } \\
\text { hsa-miR-548a-5p, hsa-miR-548b-5p } \\
\text { hsa-miR-548c-5p, hsa-miR-548d-5p } \\
\text { hsa-miR-568, hsa-miR-580, hsa-miR-590-3p, hsa-miR-634, hsa-miR-655, } \\
\text { hsa-miR-889 \& hsa-miR-96 }\end{array}$ & $\begin{array}{l}\text { E2FI, E2F2, E2F3, E2F4, E2F5, E2F6, E2F7, EGRI, } \\
\text { EP300, ESRI } \\
\text { GABPA, HDACI, HDAC2, MEF2A } \\
\text { NFYA, PAX6, PBXI, PIASI, RBI, } \\
\text { RBBP4 \& SPI }\end{array}$ \\
\hline 53 & MAPK 3 & hsa-miR-429, hsa-miR-I32, hsa-miR-2I2, hsa-miR-497 \& hsa-miR-6I3 & $\begin{array}{l}\text { AR, BCL3, CEBPB, ELKI, ELK4, } \\
\text { ESRI,ETSI,ETS2,ETV6, ETV7, FOS, GATAI, } \\
\text { GATA4, GTF2I, HDAC4, HIFI A, HSFI, HSF4, } \\
\text { JUN, JUND, MAX, MITF, MYC, NR3CI, PPARA, } \\
\text { SMADI, SMAD2, SMAD3, SMAD4, SMAD5, } \\
\text { SMAD9, SNAI2, SPI, SPIB, SREBFI, SREBF2, STAT3, } \\
\text { STAT5A, TALI, TAL2, TCF3, TGIFI, TOP2B, TP53, } \\
\text { UBTF, USFI,YYI \& ZNF7 }\end{array}$ \\
\hline 54 & PRKCA & $\begin{array}{l}\text { hsa-miR-200b, hsa-miR- I47, hsa-miR-I83, hsa-miR- I86, hsa-miR-200c, } \\
\text { hsa-miR-203, hsa-miR-205, hsa-miR-25, hsa-miR-340, hsa-miR-362-5p, } \\
\text { hsa-miR-495, hsa-miR-522 } \\
\text { hsa-miR-545, hsa-miR-548a-5p, hsa-miR-548b-5p, hsa-miR-548c-5p } \\
\text { hsa-miR-548d-5p, hsa-miR-590-3p } \\
\text { hsa-miR-603, hsa-miR-9 \& hsa-miR-I5a }\end{array}$ & $\begin{array}{l}\text { AR, BTG2, CREM, DDX5, DLX3, EDFI, EWSRI, } \\
\text { HABP4, HANDI } \\
\text { HAND2, HESI, HMGNI, HMGN2 } \\
\text { MAX, MXII::CLEC5A, MYODI } \\
\text { NFATCI, NFKBI, PA2G4, PPARA } \\
\text { RARA, RARA, SPI,TFAP2A } \\
\text { TP53, TRIM29 \& USFI }\end{array}$ \\
\hline 55 & FOS & 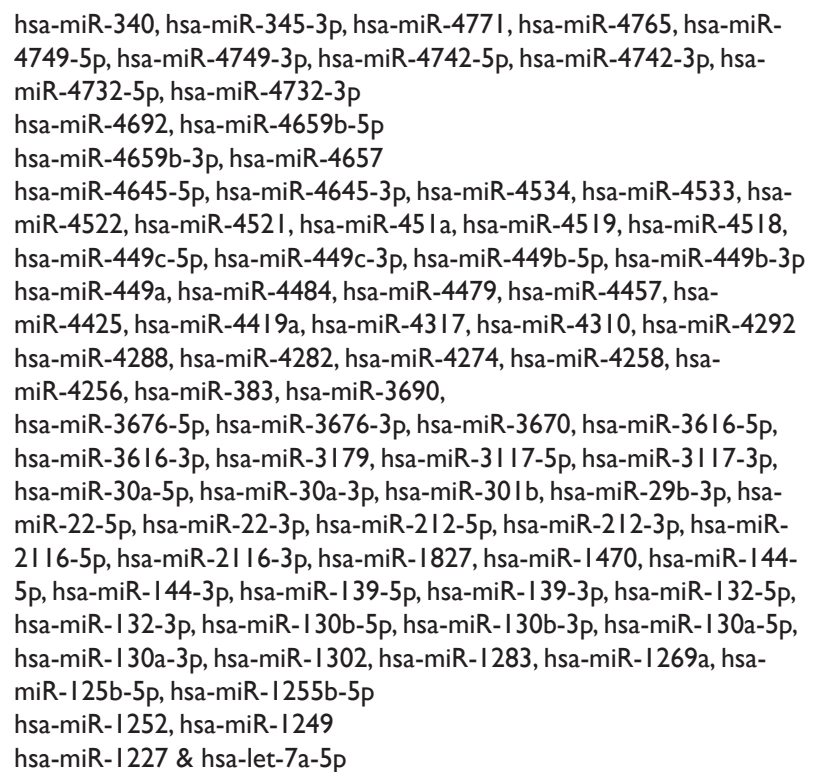 & $\begin{array}{l}\text { ATFI, ATF2, ATF7, BACH2, BATF, BCL3, CEBPB, } \\
\text { CEBPG, COBRAI, CREBI, DDIT3, EGRI } \\
\text { EGR2, ELKI, ELK3, ELK4, ESRI } \\
\text { ESR2, ETSI, ETS2, ETV4 \& FLII }\end{array}$ \\
\hline
\end{tabular}


Table Continued

\begin{tabular}{|c|c|c|c|}
\hline S. No. & Genes (Varse) & miRNAs (pharmacomiR) & Transcription factor (RegNetworks) \\
\hline 56 & JUN & $\begin{array}{l}\text { hsa-miR-324-3p, hsa-miR-I 39-5p, hsa-miR-I4I, hsa-miR-I5a, hsa- } \\
\text { miR-I6, hsa-miR-200a, hsa-miR-200b, hsa-miR-200c, hsa-miR-2 I6b, } \\
\text { hsa-miR-297, hsa-miR-30a, hsa-miR-340, hsa-miR-429, hsa-miR-495, } \\
\text { hsa-miR-496, hsa-miR-5 I 7c, hsa-miR-520d-5p, hsa-miR-522, hsa-miR- } \\
\text { 524-5p, hsa-miR-6I 2, hsa-miR-642, hsa-miR-758, hsa-miR-890, hsa- } \\
\text { miR-889, hsa-miR-802, hsa-miR-766-5p, hsa-miR-766-3p, hsa-miR-66I, } \\
\text { hsa-miR-645, hsa-miR-6 I4, hsa-miR-5706, hsa-miR-5684, hsa-miR- } \\
\text { 5587-5p, hsa-miR-5587-3p, hsa-miR-5586-5p, hsa-miR-5586-3p, hsa- } \\
\text { miR-553, hsa-miR-548z, hsa-miR-548ao-3p, hsa-miR-548aj-3p, hsa-miR- } \\
\text { 544a, hsa-miR-539-3p, hsa-miR-520b, hsa-miR-5I 9a-3p, hsa-miR-5 I 8e- } \\
\text { 5p, hsa-miR-5 I8e-3p, hsa-miR-5 I8a-5p, } \\
\text { hsa-miR-5 I8a-3p, hsa-miR-5I6a-5p, } \\
\text { hsa-miR-5 I6a-3p, hsa-miR-5008-5p, } \\
\text { hsa-miR-5008-3p, hsa-miR-5000-5p, hsa-miR-5000-3p, hsa-miR-487b, } \\
\text { hsa-miR-487a, hsa-miR-4792, } \\
\text { hsa-miR-4784, hsa-miR-477I, hsa-miR-4763-5p, hsa-miR-4763-3p, } \\
\text { hsa-miR-4732-5p, hsa-miR-4732-3p } \\
\text { hsa-miR-47I6-5p, hsa-miR-47I 6-3p } \\
\text { hsa-miR-4696, hsa-miR-4692 } \\
\text { hsa-miR-4687-5p, hsa-miR-4687-3p } \\
\text { hsa-miR-4683, hsa-miR-4665-5p } \\
\text { hsa-miR-4665-3p, hsa-miR-4659b-5p, hsa-miR-4659b-3p, hsa-miR- } \\
\text { 4640-5p, hsa-miR-4640-3p, hsa-miR-4534, hsa-miR-452I, hsa-miR-45 I a, } \\
\text { hsa-miR-45 I9, hsa-miR-449c-5p, hsa-miR-449c-3p, hsa-miR-449b-5p, } \\
\text { hsa-miR-449b-3p, hsa-miR-449a, hsa-miR-4457, hsa-miR-4434 \& hsa- } \\
\text { miR-4425 }\end{array}$ & $\begin{array}{l}\text { ABLI, AR, ATFI,ATF2, ATF3, } \\
\text { ATF4,BATF,BCL3,BCL6,BRCAI, } \\
\text { COBRAI,COPS5,CREBI,CREB5 } \\
\text { CREBBP,DDIT3,E2FI,E2F4, } \\
\text { EGRI,ELF3,ENI,EP300,ERG, ESRI, ESR2, ETSI, } \\
\text { ETS2, FOS } \\
\text { FOSLI, FOSL2, GATAI, GATA2 } \\
\text { GATA3, GTF2B, GTF2F2, HCFCI } \\
\text { HDAC3, HHEX, HIFIA, HMGAI } \\
\text { HOXA9, HOXC8, LEFI, MAF } \\
\text { MAFB, MEF2A, MYBBPIA } \\
\text { MYC, MYODI, NCOAI, NCOA2 } \\
\text { NCOA3, NFE2LI, NFE2L2, NFIC } \\
\text { NFYA, NR3CI, NR5AI, NRIPI } \\
\text { PGR, PIASI, POUIFI, RARA, RARB, RARG, RBI, } \\
\text { RBM39 } \\
\text { REL, RELA, RUNXI, RUNX2 } \\
\text { SKI, SMAD2, SMAD3, SMAD4 } \\
\text { SMARCDI, SMARCD3, SNAPC5 } \\
\text { SOXI0, SOX8, SPI, SPII, SPIB } \\
\text { SREBFI, SREBF2, STATI, STAT2 } \\
\text { STAT3, STAT4, STAT5A, STAT6 } \\
\text { TAFI,TBP,TCF20, TCF4, TCF7 } \\
\text { TCF7LI, TCF7L2, TGIFI, TRIP4 } \\
\text { TSC22D3,VDR, HSF2 \& JDP2 }\end{array}$ \\
\hline 57 & MAPK8 & $\begin{array}{l}\text { hsa-miR-26a, hsa-miR-103, hsa-miR-107, hsa-miR-| 96b, hsa-miR-2 I4, } \\
\text { hsa-miR-548c-3p \& hsa-miR-64I }\end{array}$ & $\begin{array}{l}\text { ATF2, COPS2, ELKI, ELK3, FOS HSFI, HSF4, JDP2, } \\
\text { JUN, JUNB, JUND, IL8, MECOM, MYC, NFATC2, } \\
\text { NFATC4, NR3CI, PAX2 } \\
\text { PGR, PPARG, REL, SMAD2, SMAD3, SPIB, STAT3, } \\
\text { TP53,TP73 } \\
\text { \& XRCC6 }\end{array}$ \\
\hline 58 & SRC & $\begin{array}{l}\text { hsa-miR-I297, hsa-miR-I37, hsa-miR-I4I, hsa-miR-I44, hsa-miR-I49, } \\
\text { hsa-miR-I53, hsa-miR-200a, hsa-miR-203, hsa-miR-220c, hsa-miR-3 I, } \\
\text { hsa-miR-34a, hsa-miR-34c-5p, hsa-miR-449a, hsa-miR-449b, } \\
\text { hsa-miR-9, hsa-miR-940, hsa-miR-I45 \& hsa-miR-I43 }\end{array}$ & $\begin{array}{l}\text { ABLI,AR, ARHGAP35, CTNNBI, DAB2, ESRI, } \\
\text { ESR2, ETSI, ETS2, FOXOI, GTF2I, HCLSI, } \\
\text { HNFIA, } \\
\text { HNRNPK, IKBKB, KHDRBSI, MAX, } \\
\text { MXII::CLEC5A, MYC, } \\
\text { NFKBIA, NRII2, PAX5, PGR, } \\
\text { PPARD, RXRA, SMARCBI, SMARCEI, SPI, SRF, } \\
\text { STATI, } \\
\text { STAT3, STAT5A, STAT5B, } \\
\text { STAT6, TFAP2A, THRA, TRIP6 } \\
\text { WBPII \&YTHDCI }\end{array}$ \\
\hline 59 & GRB2 & $\begin{array}{l}\text { hsa-miR-26b, hsa-miR-I24, hsa-miR-I28a, hsa-miR-I28b, hsa-miR-I4I, } \\
\text { hsa-miR-I53, hsa-miR-I 5a, hsa-miR-I5b, hsa-miR-I6, hsa-miR-I82, hsa- } \\
\text { miR-I95, hsa-miR-200a } \\
\text { hsa-miR-208b, hsa-miR-220c, hsa-miR-27a, hsa-miR-27b, hsa-miR-320, } \\
\text { hsa-miR-329, hsa-miR-376c } \\
\text { hsa-miR-424, hsa-miR-433, hsa-miR-497, hsa-miR-506, hsa-miR-520g, } \\
\text { hsa-miR-520h, hsa-miR-532-3p, hsa-miR-94I \& hsa-miR-96 }\end{array}$ & $\begin{array}{l}\text { ABLI, DAB2, DTXI, EGRI, ELKI, } \\
\text { ESRI, FOXA2, FOXOI, FOXO3, FOXO3B, } \\
\text { GABPA, HTT, KHDRBSI, KHDRBS2, MTAI } \\
\text { MTA3, NPMI, PAX5 \& TP63 }\end{array}$ \\
\hline 60 & PIK3RI & $\begin{array}{l}\text { hsa-miR-376c, hsa-miR-I03, hsa-miR-I07, hsa-miR-I 28, hsa-miR-I32, } \\
\text { hsa-miR-I36, hsa-miR-I50, hsa-miR-I 53, hsa-miR-I55, hsa-miR-I5a, } \\
\text { hsa-miR-I5b, hsa-miR-I6 } \\
\text { hsa-miR-I86, hsa-miR-I95, hsa-miR-2I, hsa-miR-2I2, hsa-miR-2I8 } \\
\text { hsa-miR-22I, hsa-miR-222, hsa-miR-29a, hsa-miR-29b, hsa-miR-29c, } \\
\text { hsa-miR-36I-5p, hsa-miR-424 } \\
\text { hsa-miR-448, hsa-miR-455-3p, hsa-miR-486-5p, hsa-miR-488, hsa- } \\
\text { miR-495, hsa-miR-497, hsa-miR-503, hsa-miR-520a-5p, hsa-miR-525-5p, } \\
\text { hsa-miR-532-3p, hsa-miR-542-3p, hsa-miR-545, hsa-miR-548a-3p, hsa- } \\
\text { miR-548c-3p, hsa-miR-579, hsa-miR-590-5p, hsa-miR-603, hsa-miR-630, } \\
\text { hsa-miR-655, hsa-miR-656, hsa-miR-767-5p, hsa-miR-89Ib } \\
\text { hsa-miR-93 \& hsa-miR-96 }\end{array}$ & $\begin{array}{l}\text { ABLI, CTCF, CTNNBI, EBFI, EGRI, EGR2, EGR3, } \\
\text { ELKI, ESRI, GABPA, GATA3, GTF2HI, HNFIA, } \\
\text { HOXA9, IRFI, KHDRBSI } \\
\text { KLFI2, MEF2A, MEISI, NFE2LI } \\
\text { NFIL3, NFKBIA, NFYA, NFYB } \\
\text { NFYC, NR2F2, PBXI, PGR, POU3FI, POU3F2, } \\
\text { RBI, SPI, USFI } \\
\text { \&WBPI I }\end{array}$ \\
\hline
\end{tabular}


Table Continued

$\begin{array}{lll}\text { S. No. Genes (Varse) miRNAs (pharmacomiR) } & \text { Transcription factor (RegNetworks) }\end{array}$

6I $\quad A B C G 2$

62

63 SOSI

hsa-miR-496, hsa-miR-192, hsa-mir-328, hsa-miR-328, hsa-miR-519c-3p hsa-miR-5I9d, hsa-miR-520a-3p hsa-miR-520b, hsa-miR-520c-3p hsa-miR-520d-3p, hsa-miR-520e hsa-miR-520f, hsa-miR-520g \& hsa-miR-520h

hsa-miR-I 86, hsa-miR-I 24, hsa-miR-I 27I, hsa-miR-I 27-5p, hsa-miR| 40-3p, hsa-miR-| 40-5p, hsa-miR- | 98, hsa-miR-204, hsa-miR-2 I I, hsa-miR-219, hsa-miR-219-5p hsa-miR-330-5p, hsa-miR-452, hsa-miR-506, hsa-miR-5I8e, hsamiR-568, hsa-miR-572, hsa-miR-650, hsa-miR-9 \& hsa-miR-96

hsa-miR-495, hsa-miR-I 24, hsa-miR-I 32, hsa-miR-| 48b, hsa-miR-I 52 hsa-miR-2II, hsa-miR-2I 2, hsa-miR-2I 7, hsa-miR-302c, hsa-miR-30a, hsa-miR-30b, hsa-miR-30d, hsa-miR-30e, hsa-miR-506, hsa-miR-5I9a, hsa-miR-5 I 9b-3p, hsa-miR-5 I 9c-3p, hsa-miR-520a-3p, hsa-miR-520b, hsa-miR-520c-3p, hsa-miR-520d-3p, hsa-miR-520e, hsa-miR-520f, hsamiR-9

\& hsa-miR-96

hsa-miR-653, hsa-miR-I03, hsa-miR-I24, hsa-miR-I 28a, hsa-miR-I28b, hsa-miR-I4I, hsa-miR-I8Ia

hsa-miR-I8Ib, hsa-miR-I8Ic

hsa-miR-I8Id, hsa-miR-I82, hsa-miR-200a, hsa-miR-223, hsa-miR-25, hsa-miR-27a, hsa-miR-27b, hsa-miR-32, hsa-miR-34b*, hsa-miR-363, hsa-miR-367, hsa-miR-433, hsa-miR-5 I 9b-3p, hsa-miR-526b hsa-miR-543, hsa-miR-552, hsa-miR-576-5p, hsa-miR-92a, hsa-miR-92b, hsa-miR-944 \& hsa-miR-96

hsa-miR-543, hsa-miR-I0I, hsa-miR-I29, hsa-miR-I 29-5p, hsa-miR-I49, hsa-miR-I82, hsa-miR-200b, hsa-miR-2 I8, hsa-miR-22I, hsa-miR-30a hsa-miR-30a-5p, hsa-miR-30b, hsa-miR-30c, hsa-miR-30d hsa-miR-30e, hsa-miR-34a, hsa-miR-429, hsa-miR-455-5p, hsa-miR-874 \& hsa-miR-96

hsa-miR-4I0, hsa-miR-2|4 \& hsa-miR-346

hsa-miR-297, hsa-miR-33 I-3p, hsa-miR-34a, hsa-miR-34b, hsa-miR-34c5p, hsa-miR-484, hsa-miR-629 hsa-miR-885-3p \& hsa-miR-939

69

PTK2B

hsa-miR-345, hsa-miR-5 I5-5p hsa-miR-I 55, hsa-miR-I8Ia, hsa-miR-I8Ib, hsa-miR-I8Id, hsa-miR-204

Transcription factor (RegNetworks)

FOS, JUN, MAX \& USFI

ABLI, BCL3, ELKI, EP300, ESR I

ESRI, FOS, GABPA, JUN, NPMI POU2FI, REL, RELA, SPI, STAT5A, STAT5B, TOPORS

\& TP53

AR, BINI, CTCF, EGRI, ESRI, NFIC, RARA, SPI, STAT3, TFAP2A, USFI, NFIC, RARA, SPRYI, SPRY2 CCNDI, SPRY4

SPRY3, BCL2LI, CCND2, CCND3

MYC, SPREDI \& SPRED2

ABLI,AHR, ARNT, ATFI, ATM, CEBPA, CEBPB, CIITA, CREBBP, CREM, EDFI, EGRI, EP300, FHL5, GLI2, GTF2F2, KAT5, KCNIP3, MYC, NFKBI, NFKB2,

NR3CI, PDXI, REL, RELA, RELB,

SMARCA4, SMARCA5, SOX9,

SRF, STAT3,THRA \& TLX2

ABLI, ATF2, CREBI, CTCF, E2F4

EEFIAI, EGRI, ESRI, GTF2HI

KHDRBSI, NFYA, SREBFI, SREBF2, TOPORS,

USFI \&YYI

EP300, FOS, JUN, SLA2, STAT5A

STAT5B \& YY

ABLI, ATF6, CTCF, HNF4A, NFYA, NR2FI, NR2F2, SLA2, USFI \& XBPI

AIP,APEXI,ARNT, ATFI,ATF2

BDPI, BRCAI, BRFI, CREBBP,

CREM, CTDPI, CTNNBI, CUX2,

DDIT3, EGRI, ETSI, FOS, GTF2AI, GTF2AIL, HCLSI, HDACI, HDAC2, HDAC3, HES6,

HIFIA, HMGAI, HMGA2, HOXB7

HSFI, IFII 6, IRF2, JUN, KLFI, MAX, MAZ, MEF2C, MYB, MYC,

MYCN, MYF5, NAPIL4, NFIC,

NFKBI, NFKBIA, NKX2-5, PGR,

PML, RELA, SHOX, SPI, SPIB,

SRF, SSRPI, SUBI, TCF7L2, TOPORS, TP53, UBTF,

VDR,YYI

$\& \mathrm{ZHXI}$

CTCF, EGRI, EWSRI, GABPA

hsa-miR-5 I9c-3p, hsa-miR-I9a, hsa-miR-205, hsa-miR-23a, hsa-miR-23b, HNF4A, MYC, NFKBI, REST

hsa-miR-647, hsa-miR-892b \& hsa-miR-940

\& TP53 
Table Continued

\begin{tabular}{|c|c|c|c|}
\hline S. No. & Genes (Varse) & miRNAs (pharmacomiR) & Transcription factor (RegNetworks) \\
\hline 70 & CAMK2B & hsa-miR-544 & $\begin{array}{l}\text { AHR, ARNT, ATF4, CTCF, E2FI } \\
\text { E2F2, E2F3, E2F4, E2F5, E2F6 } \\
\text { E2F7, EGRI, EGR2, EGR3, ETSI } \\
\text { MEF2A, NFE2LI, PAX5, RORA } \\
\text { RREBI, RXRG, SPI, THRB, USFI } \\
\text { \& XBPI }\end{array}$ \\
\hline 71 & HBXIP & $\begin{array}{l}\text { hsa-miR-192, hsa-miR-106a, hsa-miR-I06b, hsa-miR-I40-5p, hsa- } \\
\text { miR-I45, hsa-miR-16, hsa-miR-I7, } \\
\text { hsa-miR-I8a, hsa-miR-I8b, hsa-miR-20a, hsa-miR-20b, hsa-miR-370, } \\
\text { hsa-miR-378, hsa-miR-492, hsa-miR-496, hsa-miR-5 I 5-3p, hsa-miR- } \\
\text { 5I9d, hsa-miR-5 I9e, hsa-miR-520d-5p, hsa-miR-520g, hsa-miR-520h, } \\
\text { hsa-miR-524-5p, hsa-miR-545, hsa-miR-590-3p, hsa-miR-606, hsa- } \\
\text { miR-6I9, hsa-miR-63I, hsa-miR-876-3p \& hsa-miR-93 }\end{array}$ & $\begin{array}{l}\text { EEFIAI, GABPA, HNFIA, HNF4A MAFI, MAX, } \\
\text { POU2FI, USFI \& } \\
\text { ZBTBI } 6\end{array}$ \\
\hline 72 & PPP3CA & $\begin{array}{l}\text { hsa-miR-328, hsa-let-7a, hsa-let-7b } \\
\text { hsa-let-7c, hsa-let-7e, hsa-let-7f, } \\
\text { hsa-let-7g, hsa-let-7i, hsa-miR-I00, hsa-miR-I06a, hsa-miR-I06b, hsa- } \\
\text { miR-I42-3p, hsa-miR-I45, hsa-miR-I53, hsa-miR-I7, hsa-miR-I82, } \\
\text { hsa-miR-20b, hsa-miR-2I, hsa-miR-300 } \\
\text { hsa-miR-30a, hsa-miR-30a-5p, hsa-miR-30b, hsa-miR-30c, hsa-miR-30d, } \\
\text { hsa-miR-30e, hsa-miR-3I, hsa-miR-340, hsa-miR-34b, hsa-miR-372, } \\
\text { hsa-miR-38I, hsa-miR-4I0, hsa-miR-495, hsa-miR-499-5p, hsa-miR-50I- } \\
\text { 3p,hsa-miR-502-3p, hsa-miR-5 I9a, hsa-miR-5I9b-3p, hsa-miR-5 I9c-3p, } \\
\text { hsa-miR-520d-3p, hsa-miR-520d-5p, hsa-miR-520e, hsa-miR-520g, hsa- } \\
\text { miR-520h, hsa-miR-524-5p, hsa-miR-590-5p, hsa-miR-635, hsa-miR-93, } \\
\text { hsa-miR-96 } \\
\text { hsa-miR-98 \& hsa-miR-99a }\end{array}$ & $\begin{array}{l}\text { AHR, ARNT, CEBPB, CUXI, E2FI } \\
\text { E2F2, E2F3, E2F4, E2F5, E2F6, E2F7, EGRI, FOXCI, } \\
\text { FOXJ2, NFAT5, NFATCI, NFATC2, NFATC3, } \\
\text { NFATC4, NFYA, PPARG \& ZBTB6 }\end{array}$ \\
\hline 73 & NFATCI & $\begin{array}{l}\text { hsa-miR-5 I9c, hsa-miR-I22, hsa-miR-I24, hsa-miR-I37, hsa-miR-I43, } \\
\text { hsa-miR-I55, hsa-miR-506 \& hsa-miR-6II }\end{array}$ & $\begin{array}{l}\text { AHR, ARNT,ATF2, CDC5L, EP300, FOXCI, } \\
\text { FOXF2, FOXII, FOXOI, FOXO3, FOXO3B, } \\
\text { FOXO4, NFKBI, PAX5, REL, RELA, SPII, SRF \& } \\
\text { USFI }\end{array}$ \\
\hline 74 & РPРЗСВ & $\begin{array}{l}\text { hsa-miR-37I-5p, hsa-miR-I0I, hsa-miR-I03, hsa-miR-I07, hsa- } \\
\text { miR-I 297, hsa-miR-I37, hsa-miR-I40-3p } \\
\text { hsa-miR-I 5a, hsa-miR-I5b, hsa-miR-I6, hsa-miR-I92, hsa-miR-I95 } \\
\text { hsa-miR-I96b, hsa-miR-I9b, hsa-miR-2I5, hsa-miR-222, hsa-miR-26a, } \\
\text { hsa-miR-26b, hsa-miR-30a, hsa-miR-30a-5p, hsa-miR-30b, hsa-miR-30e, } \\
\text { hsa-miR-326, hsa-miR-424, hsa-miR-506, hsa-miR-559, hsa-miR-6I3, } \\
\text { hsa-miR-645, hsa-miR-646, hsa-miR-660, hsa-miR-768-3p \& hsa- } \\
\text { miR-802 }\end{array}$ & $\begin{array}{l}\text { CEBPA, CEBPB, DDIT3, EGRI, } \\
\text { EP300, NFAT5, NFATCI, NFATC2 } \\
\text { NFATC3, NFATC4, NR3CI \& PAX2 }\end{array}$ \\
\hline 75 & RCANI & $\begin{array}{l}\text { hsa-miR-I 48a, hsa-let-7b, hsa-miR - 103, hsa-miR-107, hsa-miR-124, hsa- } \\
\text { miR-323-3p, hsa-miR-34a, hsa-miR-34c-5p \& hsa-miR-506 }\end{array}$ & $\begin{array}{l}\text { ALXI, CREBI, EBFI, EGRI, IRFI } \\
\text { JUN, MAX, POU3FI, SPI, SPII } \\
\text { STAT5A, TGIFI, USFI \&VSX2 }\end{array}$ \\
\hline 76 & PPARG & $\begin{array}{l}\text { hsa-miR-I 96a, hsa-miR-I28a, hsa-miR-I30a, hsa-miR-I 30b, hsa- } \\
\text { miR-I 38, hsa-miR-I44, hsa-miR-20b, hsa-miR-27a, hsa-miR-27b, hsa- } \\
\text { miR-30I, hsa-miR-338-5p, hsa-miR-34a, hsa-miR-409-3p, hsa-miR-449a, } \\
\text { hsa-miR-520b, hsa-miR-520c-3p, hsa-miR-520d-3p, hsa-miR-548a-5p, } \\
\text { hsa-miR-548b-5p, hsa-miR-548c-5p, hsa-miR-548d-5p \& hsa-miR-559 }\end{array}$ & $\begin{array}{l}\text { AR, BRD8, CEBPA, CEBPB, COPS5, CREBI, EDFI, } \\
\text { EGRI, ESRI, ESR2, FOXOI, GATAI, HDAC3, } \\
\text { HMGAI, MEDI, NCOAI } \\
\text { NCOA2, NCOA3, NCOA4, NCOA6 } \\
\text { NCORI, NCOR2, NFE2LI, NFE2L2, NROBI \& } \\
\text { NROB2 }\end{array}$ \\
\hline 77 & NOS3 & hsa-miR-IOb & $\begin{array}{l}\text { BACHI, CTCF, EPASI, ERG, ESRI, ESR2, ETSI, } \\
\text { ETV4, GABPA } \\
\text { GATAI, GATA2, GATA3, JUN, NR2F2, RORA, SPI, } \\
\text { SPI, SP3, SPII, TALI ::GATA I, TFAP2A \& TP53 }\end{array}$ \\
\hline 78 & ADRB2 & $\begin{array}{l}\text { hsa-miR-I 42-3p, hsa-let-7a, hsa-let-7b, hsa-let-7c, hsa-let-7d, hsa-let-7e } \\
\text { hsa-let-7f, hsa-let-7g, hsa-let-7i, hsa-miR-I55, hsa-miR-I5a, hsa-miR-I5b, } \\
\text { hsa-miR-16, hsa-miR-195, hsa-miR-2 I 6b, hsa-miR-27a, hsa-miR-30a, } \\
\text { hsa-miR-30a-5p, hsa-miR-30b } \\
\text { hsa-miR-30d, hsa-miR-30e, hsa-miR-424, hsa-miR-497, hsa-miR-556-3p, } \\
\text { hsa-miR-605, hsa-miR-607 } \\
\text { hsa-miR-625, hsa-miR-634, hsa-miR-7, hsa-miR-888 \& hsa-miR-98 }\end{array}$ & $\begin{array}{l}\text { CEBPA, CEBPB, CREBI, CUXI } \\
\text { ELKI, GATAI, HLF, NFIC, NFIL3 } \\
\text { PBXI, RORA, STAT5A \& TBP }\end{array}$ \\
\hline
\end{tabular}




\begin{tabular}{|c|c|c|}
\hline S. No. & Genes (Varse) & miRNAs (pharmacomiR) \\
\hline 79 & CORIN & $\begin{array}{l}\text { hsa-miR-873, hsa-miR-I06a, hsa-miR-I06b, hsa-miR-I38, hsa-miR-I48a, } \\
\text { hsa-miR-I52, hsa-miR-I7, hsa-miR-20a, hsa-miR-20b, hsa-miR-302a, } \\
\text { hsa-miR-302b, hsa-miR-302c, hsa-miR-302d, hsa-miR-373, hsa-miR-495, } \\
\text { hsa-miR-520a-3p, hsa-miR-520b, hsa-miR-520c-3p } \\
\text { hsa-miR-520d-3p, hsa-miR-520e } \\
\text { hsa-miR-520f, hsa-miR-548a-3p, hsa-miR-56I, hsa-miR-582-3p \& hsa- } \\
\text { miR-93 }\end{array}$ \\
\hline 80 & GNAS & $\begin{array}{l}\text { hsa-miR-I39-5p, hsa-let-7i, hsa-miR-I05, hsa-miR-I36, hsa-miR-I38, } \\
\text { hsa-miR-I43, hsa-miR-I55, hsa-miR-I5b, hsa-miR-I 8a, hsa-miR-205, } \\
\text { hsa-miR-2I8, hsa-miR-2I9-2-3p, hsa-miR-2I9-5p, hsa-miR-22II, hsa- } \\
\text { miR-223, hsa-miR-338-3p, hsa-miR-34b, hsa-miR-36I-3p, hsa-miR- } \\
\text { 36I-5p, hsa-miR-362-5p, hsa-miR-452, hsa-miR-453, hsa-miR-489, } \\
\text { hsa-miR-494, hsa-miR-502-5p, hsa-miR-5 I 2-3p, hsa-miR-532-5p, hsa- } \\
\text { miR-582-3p, hsa-miR-588, hsa-miR-596, hsa-miR-624, hsa-miR-625, hsa- } \\
\text { miR-629, hsa-miR-630, hsa-miR-660, hsa-miR-876-3p \& hsa-miR-943 }\end{array}$ \\
\hline
\end{tabular}

HSP90AAI

hsa-miR-9, hsa-miR-I42-3p, hsa-miR-196a, hsa-miR-196b, hsa-miR-25, hsa-miR-32, hsa-miR-493 \& hsa-miR-92a

hsa-miR-| 45, hsa-miR-I24, hsa-miR-I55, hsa-miR-200b, hsa-miR-200c, hsa-miR-22I, hsa-miR-222, hsa-miR-300, hsa-miR-36I-5p, hsa-miR-38I, hsa-miR-429, hsa-miR-494, hsa-miR-506, hsa-miR-654-5p \& hsa-miR-944

hsa-miR-384, hsa-miR-I, hsa-miR- I24, hsa-miR- I37, hsa-miR-|4I, hsamiR-142-3p, hsa-miR-I44, hsa-miR-18Ia, hsa-miR-I8Ib, hsa-miR-I8Ic, hsa-miR-I8Id, hsa-miR-I82, hsa-miR-I83, hsa-miR-I8a, hsa-miR-I8b, hsa-miR-200a, hsa-miR-200b, hsa-miR-200c, hsa-miR-204 hsa-miR-206, hsa-miR-2I I, hsa-miR-22, hsa-miR-29a, hsa-miR-29b hsa-miR-29c, hsa-miR-300, hsa-miR-30a, hsa-miR-30b, hsa-miR-320, hsa-miR-328, hsa-miR-340, hsa-miR-377, hsa-miR-38I, hsa-miR-4I 0 , hsa-miR-429, hsa-miR-506, hsa-miR-5 I 5-5p, hsa-miR-5 I 8d-5p, hsamiR-543, hsa-miR-548c-3p, hsa-miR-582-5p, hsa-miR-603 \& hsamiR-889

hsa-miR-28-5p , hsa-miR-2। 4, hsa-miR-542-5p \& hsa-miR-873 hsa-miR-598

hsa-miR-98, hsa-miR-I24, hsa-miR-2 19-5p, hsa-miR-27a, hsa-miR-27b, hsa-miR-506, hsa-miR-626, hsa-miR-876-5p \& hsa-miR-9
Transcription factor (RegNetworks)

CTCF

CEBPA, CTCF, CUXI, E2F2, E2F4

HSFI, NFIL3, REST,TFAP2A, TFAP2C, USFI, VSX2

\&YYI

AHR, AIP,AR, ARNT, ARNTL, CREBI, CTCF, EGR I, EIF2AK2, ESR I, HIF I A, HSFI, HSF2, IKBKB, IKBKG, MAX, MXII::CLEC5A, MYC, MYODI, NPAS2, NR2C2, NR3CI, NR3C2, PPARA, REST, RUNXITI, SRF, STATI, STAT3, TFAP2A, TFAP2C TP53, USFI \&YYI

ARNT, BACH2, BCL3, ELKI, EP300, ESR I, GTF2A2, GTF2EI, GTF2E2, GTF2FI, MAX, MXII::CLEC5A, MYC,TAFI,TAF4,TBP,TBPLI, TRIP4 \& USF

AES, E2F2, ERCC3, GTF2AI, GTF2A2, GTF2B, GTF2E2, GTF2F2, GTF2HI, GTF2H4, NFYA SNDI, SPI,TAF6,TBP,TCEAI,TRIM24,YYI \& ZEBI

AHR, AR, ARNT, ATFI, ATF2, ATF3, ATF4, ATF5, ATF6, ATF7,

CEBPA, CEBPB, CREBI, CREBBP, CTCF, DDX54, E2FI, ECD, EGRI, EGR2, EGR3, ETSI, ETS2, HIFIA, JUN, KDM5A, MEDI4, NCOAI, NCOA2, NCOA6

NCORI, NCOR2, NFIA, NFIC, NFKBI, NFKB2,NR2F2 \& NR2F6

ABLI,ALXI, ATF2, BARDI, BRCAI, CREBI, CREBBP, CTCF, CTDPI, ELL, ERCC6, FUS, GTF2B, HTATSFI, KLFI2, MCM3, MED2I, NFIL3, NONO, NR3CI, POLR2C POLR2D, POLR2E, POLR2F, POLR2G, POLR2H, POLR2L, POU2FI, PQBPI, SAFB, SNDI, SPI, STATI, STAT3, SUPT5H, TAFI0,TBP,TCEAI, TCERGI,TFAP2A, UPFI, USF XAB2, XRCC5,YYI \& ZNF74

CREBI, EGRI, EGR2, EP300, HDAC5, MZFI, NFKBI, PATZI

RELA, SPI \& VSX2

BCL3, BRD8, COPS2, CTNNBI, EDFI, EGRI, ESRI, FOXOI, FUS, GATA2, HMGA I, JMIDIC, MEDI, MYC, MYODI, NCOAI, NCOA2, NCOA3, NCOA4, NCOA6, NCOR2, NFKBI, NFKBIB, NPAS2, NROB2, NRIH2, NRIH3, NRIH4, NRII2, NRII3, NR2FI, NR4A2, NRBF2, NRIPI, NSDI, PARPI, PAX8, POU2FI, PPARA, PPARD, PPARG, PPARGCIA, RARA, RARB, RARG \& RELA 
Table Continued

\begin{tabular}{|c|c|c|}
\hline S. No. & Genes (Varse) & miRNAs (pharmacomiR) \\
\hline 88 & SMARCDI & $\begin{array}{l}\text { hsa-let-7c, hsa-miR-I0I, hsa-miR-I27-5p, hsa-miR-I33a, hsa-miR-I33b, } \\
\text { hsa-miR-I4I, hsa-miR-I48a, } \\
\text { hsa-miR-I48b, hsa-miR-I50, hsa-miR-I52, hsa-miR-I9I, hsa-miR-200a, } \\
\text { hsa-miR-2I, hsa-miR-223, hsa-miR-324-5p, hsa-miR-325, hsa-miR-369- } \\
\text { 5p, hsa-miR-370, hsa-miR-42 I, hsa-miR-490-3p, hsa-miR-5I5-5p, hsa- } \\
\text { miR-552, hsa-miR-556-3p, hsa-miR-590-5p, hsa-miR-624, hsa-miR-7 \& } \\
\text { hsa-miR-940 }\end{array}$ \\
\hline 89 & SMARCEI & $\begin{array}{l}\text { hsa-let-7a, hsa-miR-I03, hsa-miR-I07, hsa-miR-I 34, hsa-miR-I35a, hsa- } \\
\text { miR-I 35b, hsa-miR-22, hsa-miR-485-3p, hsa-miR-494, hsa-miR-5I 2-5p, } \\
\text { hsa-miR-520a-5p, hsa-miR-548a-5p, hsa-miR-548b-5p, hsa-miR-548c-3p, } \\
\text { hsa-miR-548c-5p, hsa-miR-548d-5p, hsa-miR-555, hsa-miR-603, hsa- } \\
\text { miR-609, hsa-miR-760 \& hsa-miR-9 }\end{array}$ \\
\hline 90 & ANXAI & $\begin{array}{l}\text { hsa-let-7i , hsa-miR-196a, hsa-miR-22I, hsa-miR-222, hsa-miR-376c, } \\
\text { hsa-miR-598 \& hsa-miR-758 }\end{array}$ \\
\hline
\end{tabular}

hsa-let-7b, hsa-miR-I06b, hsa-miR-I24, hsa-miR-125a-3p, hsa-miR-203 hsa-miR-34b*, hsa-miR-34c-5p, hsa-miR-377, hsa-miR-409-3p, hsamiR-494, hsa-miR-506, hsa-miR-548c-3p, hsa-miR-628-5p, hsa-miR-768$3 p$ \& hsa-miR-96

hsa-let-7e, hsa-miR-I38, hsa-miR-I55, hsa-miR-27a, hsa-miR-27b, hsamiR-29a, hsa-miR-29b, hsa-miR-29c, hsa-miR-452, hsa-miR-588, hsamiR-590-3p \& hsa-miR-768-5p hsa-let-7d, hsa-miR-I45, hsa-miR-2 I6a, hsa-miR-22 I, hsa-miR-4 I 2, hsamiR-582-3p, hsa-miR-647 \& hsa-miR-648

hsa-let-7f, hsa-miR-I, hsa-miR-I0I, hsa-miR-I 32, hsa-miR-I44, hsamiR-I53, hsa-miR-I85, hsa-miR-I9a, hsa-miR-I9b, hsa-miR-203, hsa-miR-2। 2, hsa-miR-2 I 6, hsa-miR-2I6a, hsa-miR-22।, hsa-miR-222, hsa-miR-223, hsa-miR-30a, hsa-miR-30b, hsa-miR-30c, hsa-miR-30d, hsa-miR-30e, hsa-miR-3 I

hsa-miR-324-5p, hsa-miR-340, hsa-miR-377, hsa-miR-485-3p, hsa-miR486-5p, hsa-miR-548c-3p, hsa-miR-555, hsa-miR-568, hsa-miR-576-5p, hsa-miR-583, hsa-miR-625, hsa-miR-765, hsa-miR-876-3p, hsa-miR-8853p \& hsa-miR-9

hsa-miR-202, hsa-miR-I, hsa-miR-I03, hsa-miR-I07, hsa-miR-I22, hsamiR-I87, hsa-miR-190, hsa-miR-I90b, hsa-miR-I99a-3p, hsa-miR-206, hsa-miR-2।4, hsa-miR-22 hsa-miR-24, hsa-miR-26a, hsa-miR-26b, hsa-miR-338-3p, hsa-miR-384 miR-56I, hsa-miR-582-5p, hsa-miR-607 \& hsa-miR-633

hsa-miR-30e , hsa-miR-I03, has-miR-I07, hsa-miR-I0b, hsa-miR-I25a3p, hsa-miR-I28, hsa-miR-I35a, hsa-miR-I35b, hsa-miR-I 37 hsa-miR-| 44, hsa-miR-I53, hsa-miR-I5a, hsa-miR-I5b, hsa-miR-I6 hsa-miR-I82, hsa-miR-195, hsa-miR-19a, hsa-miR-19b, hsa-miR-200b, hsa-miR-200c, hsa-miR-217

hsa-miR-2 I 9-I-3p, hsa-miR-25, hsa-miR-27a, hsa-miR-27b, hsa-miR-30a, hsa-miR-30b, hsa-miR-30c, hsa-miR-30d, hsa-miR-30e, hsa-miR-338-5p, hsa-miR-340, hsa-miR-363, hsa-miR-369-3p, hsa-miR-370 hsa-miR-374a, hsa-miR-374b, hsa-miR-424, hsa-miR-448, hsa-miR-497, hsa-miR-5 I 9b-3p, hsa-miR-5 I 9c-3p, hsa-miR-543, hsa-miR-548d-3p, hsa-miR-552, hsa-miR-56I

hsa-miR-57I, hsa-miR-582-5p, hsa-miR-590-3p, hsa-miR-6I7, hsamiR-625, hsa-miR-655, hsa-miR-876-5p, hsa-miR-944 \& hsa-miR-96 hsa-miR-488, hsa-miR-5 I I, hsa-miR-520d-5p, hsa-miR-524-5p, hsa-
Transcription factor (RegNetworks)

ATFI, ATF2, ATF3, ATF4, ATF5

ATF6, ATF7, CREBI, FOS, GATAI, JUN, MAX, MEISI, MXII::CLEC5A, MYC, NR3CI\& REL

BAZIB, E2FI, E2F2, E2F3, E2F4, E2F5, E2F6, E2F7, ESR I, ESR2, IRF7, NCOA I, NCOA2, NCOA3, NR3CI, PAX5, RELB \& SMADI

FOS, FOXLI, POU3FI \& TBP

ABLI,AR, ATF6, E2FI, E2F4, EGRI, ESRI, NFIC, PAX2, RFXI

SPI,TFDPI,TP53 \& XBPI

\section{AR, ARNT,ASCC2, E2FI, E2F2,}

E2F3, E2F4, E2F5, E2F6, E2F7, ESR I, POU2FI, POU2F2, POU3FI,

POU3F2, POU3F3, POU5FI, PPARA, PPARD, PPARG, RXRA,

$R X R B$ \& RXRG

ATF7IP, EEFIAI, EGRI, EGR3, ELKI, EP300, GABPA, HNF4A, HNRNPAB, NFKBI, NFKB2, NFYA, NFYB, NFYC, POLR2A, POU3F2, PPARG, REL, RELA, SMAD3, SMAD9, SMARCA4, SPI, SRF, STATI, STAT3, TBP,TFAP2A \& USFI

ARNT, CREBI, E2FI, E2F2, E2F3, E2F4, E2F5, E2F6, E2F7, FOXD3, FOXII, GATA2, HNF4A, MAX, MLLTI, MXII::CLEC5A, MYC, MZFI, PAX5, POU2FI, POU3FI, PPARG, SMARCA4, SOX9, SPI, SRF,TFAP2A, TFAP2C, TP53, USFI \&YYI

BCL3, CREBBP, EGRI, EP300, ESRI, ESRRA, FOXF2, GTF2EI, GTF2E2, GTF2FI, GTF2F2, HSFI, IKZFI, JUN, MAX, MCM2, MXII::CLEC5A, MYC, NCOAI, NCOA3, NCOA4, NR2FI, NR5AI, POLR2A, POU2FI, POU2F2, POU3F2, REL, RELA, TAF9, TBP,TBPLI,TCEA2, THRA, USF I \&VDR

ATF2, CREBI, CTCF, JUN, PATZI PAX5, SPI,TBPLI \& TFAP2A 
Table Continued

S. No. Genes (Varse) miRNAs (pharmacomiR) Transcription factor (RegNetworks)

$97 \quad$ SMARCA4 SMARCBI
hsa-miR-30c, hsa-miR-I, hsa-miR-I0I, hsa-miR-I32, hsa-miR-I39-5p, hsa-miR-I44, hsa-miR-I55, hsa-miR-I9I, hsa-miR-206, hsa-miR-2I 2 , hsa-miR-22I, hsa-miR-222, hsa-miR-296-5p, hsa-miR-30 la, hsa-miR30c, hsa-miR-452, hsa-miR-489, hsa-miR-495, hsa-miR-503, hsa-miR508-5p, hsa-miR-5 I 2-3p, hsa-miR-5I 7b, hsa-miR-568, hsa-miR-569, hsamiR-574-3p, hsa-miR-582-5p, hsa-miR-6I3, hsa-miR-765 \& hsa-miR-942

hsa-miR-30d, hsa-miR-I, hsa-miR-192, hsa-miR-206 \& hsa-miR-300

hsa-miR-30a, hsa-let-7a, hsa-let-7b, hsa-let-7c, hsa-let-7d, hsa-let-7e, hsa-let-7f, hsa-let-7g, hsa-let-7i, hsamiR-I, hsa-miR-I24, hsa-miR-I29-5p, hsa-miR-I44, hsa-miR-I53, hsamiR-196a, hsa-miR-1 96b, hsa-miR-202, hsa-miR-206, hsa-miR-208b, hsa-miR-2 I4, hsa-miR-23a, hsa-miR-23b, hsa-miR-29b, hsa-miR-29c, hsa-miR-330-3p, hsa-miR-339-3p, hsa-miR-488, hsa-miR-506, hsa-miR548d-3p, hsa-miR-567, hsa-miR-6|0, hsa-miR-64| \& hsa-miR-98

hsa-miR-30b, hsa-miR-28-5p, hsa-miR-30a, hsa-miR-30b, hsa-miR-30c, hsa-miR-30e, hsa-miR-519a, hsa-miR-5 I 9b-3p, hsa-miR-5 I 9c-3p, hsa-miR-5I 9d, hsa-miR-568, hsa-miR-608, hsa-miR-708 \& hsa-miR-938

hsa-miR-196b, hsa-miR-192, hsa-miR-324-5p, hsa-miR-5I8a-5p, hsamiR-527, hsa-miR-583
AHR, ARIDIA, ARIDIB, BRCAI, BRWDI, CEBPB, CHD4, CREBI, CTNNBI, E2FI, E2F4, EGRI, ESRI, ETS2, GABPA, HSFI, IKZFI, MYC, MZFI, NR3CI, PAX6, PBRMI, RBI, RELB, RFXAP, SMARCBI, SMARCCI, SMARCEI, STAT2, TMFI, TP53,YYI \& ZMYNDII

CEBPB, E2F4, FOS, FOSB, FOSL I HNF4A, JUN, JUNB, JUND, KLF I MECP2, MYC, NR3CI, RBI, RELB SMARCA4,TCF3,TFAP2A, TP53 \&YEATS4

CEBPB, FOXCI, GABPA, HOXA9 KLFI, MEISI, NR3CI, RELB, SIN3A \& SMARCA4

BAZIB, CTCF, ESRI, GABPA POLR2C \& RELB

FOS, HNF4A, JUN, MAX, NR2FI, $\mathrm{NR} 3 \mathrm{CI}, \mathrm{POU} 2 \mathrm{FI}, \mathrm{POU} 2 \mathrm{~F} 2, \mathrm{POU} 3 \mathrm{FI}$ POU3F2, POU3F3, POU5FI \& TFAP2A

\section{Construction of regulatory network (cytoscape)}

The regulatory network was constructed with 101 genes, 576 miRNAs and 638 TFs. Network was initiated from the association of Pharmacogenomics and Clinical variants with the 1214 regulators (i.e. 638 TFs \& 576 miRNAs) to interact with the 101 target genes in such a way to form 1316 nodes and 4174 edges.

\section{Identification of hub genes in regulatory network in} top-down approach (cytohubba)

The genes and their regulators (Micro RNAs \& Transcription Factors) were subjected to the analysis in cytohubba by various global based statistical methods like Edge Percolated Component, Bottleneck, EcCentricity, Closeness, Radiality, Betweenness and Stress along with local based statistical methods like Maximal Clique Centrality, Density of Maximum Neighborhood Component, Maximum Neighborhood Component and degree to identify their connectivity. Among the various methods of analysis in top down approach only a global based statistics of EcCentricity method and the local based statistics of Maximal Clique Centrality along with Density of Maximum Neighborhood Component and Clustering coefficient methods in cytohubba resulted in obtaining a regulatory network of gene-miRNA-TFs in top-down approach. The details of regulatory network were given in Figure 1.

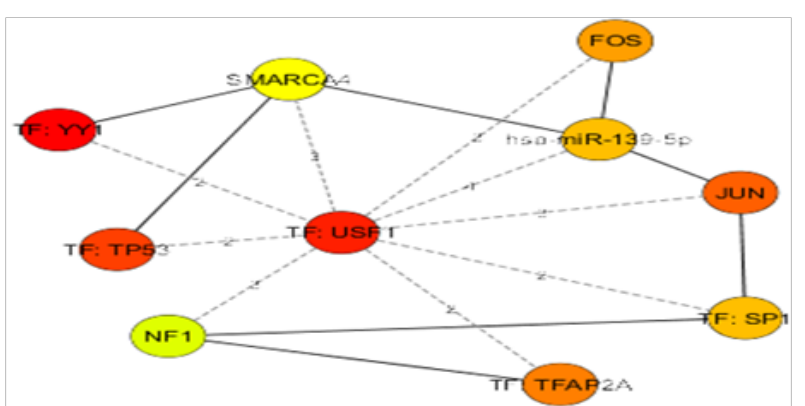

Figure I Regulatory Network of SMARCA4, FOS, JUN \& NFI (Bottleneck method).

\section{Implication of miRNAs in regulatory network}

The implication of miRNAs in the regulatory network was analyzed on the basis of compatibility with respect to gene-miRNA seed pairing and gene-miRNA-miRNA triplex with respect to nature of binding and the details were given in Table 2 .

\section{Pathway analysis}

The obtained genes from Varse data were subjected to pathway analysis in DAVID on the basis of P value and Benjamini statistic and the result is given in Table 3 . 
Table 2 Implication of miRNA in regulatory network

\begin{tabular}{|c|c|c|c|c|c|c|}
\hline S. No. & Genes & microRNA & $\begin{array}{l}\text { No. of binding Sites } \\
\text { (mIRmap) }\end{array}$ & Paired miRNA & $\begin{array}{l}\text { Binding energy in } \mathrm{Kcal} / \mathrm{mol} \text {. } \\
\text { (Triplex RNA) }\end{array}$ & $\begin{array}{l}\text { Nature of } \\
\text { binding (Triplex } \\
\text { RNA) }\end{array}$ \\
\hline $\mathrm{I}$. & FOS & & NIL & hsa-miR-543 & -26.96 & Canonical triplex \\
\hline 2. & JUN & & NIL & NIL & NIL & NIL \\
\hline 3. & NFI & hsa-miR-I39-5p & NIL & hsa-miR-590-3p & -22.46 & $\begin{array}{l}\text { Self } \\
\text { complementary }\end{array}$ \\
\hline 4. & SMARCA4 & & NIL & hsa-miR-7 & -21.16 & Canonical triplex \\
\hline
\end{tabular}

Table 3 Annotation of Kegg Pathways in direct approach

\begin{tabular}{|c|c|c|c|}
\hline S. No. & Pathway & P Value & Benjamini \\
\hline I & T cell receptor signaling pathway & 3.7IE-27 & $7.08 \mathrm{E}-25$ \\
\hline 2 & ErbB signaling pathway & $1.03 \mathrm{E}-19$ & $9.82 \mathrm{E}-18$ \\
\hline 3 & Hepatitis B & $4.00 \mathrm{E}-19$ & $2.55 \mathrm{E}-17$ \\
\hline 4 & Natural killer cell mediated cytotoxicity & $4.20 \mathrm{E}-18$ & $2.01 \mathrm{E}-16$ \\
\hline 5 & Fc epsilon RI signaling pathway & $2.93 \mathrm{E}-17$ & I.I2E-I5 \\
\hline 6 & Oxytocin signaling pathway & $4.79 \mathrm{E}-\mathrm{I} 7$ & I.53E- 15 \\
\hline 7 & Neurotrophin signaling pathway & $6.32 \mathrm{E}-17$ & $3.00 \mathrm{E}-15$ \\
\hline 8 & Osteoclast differentiation & $3.45 \mathrm{E}-16$ & $7.99 \mathrm{E}-15$ \\
\hline 9 & Estrogen signaling pathway & $7.95 \mathrm{E}-16$ & I.65E-I4 \\
\hline 10 & B cell receptor signaling pathway & I.I3E-I5 & $2.12 \mathrm{E}-14$ \\
\hline II & Focal adhesion & I.24E-I4 & $2.14 \mathrm{E}-13$ \\
\hline 12 & Choline metabolism in cancer & $2.43 \mathrm{E}-14$ & 3.87E- 13 \\
\hline 13 & Prostate cancer & $5.49 \mathrm{E}-\mathrm{I} 4$ & $8.06 \mathrm{E}-13$ \\
\hline 14 & GnRH signaling pathway & $9.23 \mathrm{E}-14$ & $1.26 \mathrm{E}-12$ \\
\hline 15 & MAPK signaling pathway & $8.75 \mathrm{E}-13$ & I.IIE-II \\
\hline 16 & Proteoglycans in cancer & $9.97 \mathrm{E}-13$ & I.I9E-II \\
\hline 17 & Prolactin signaling pathway & $1.16 \mathrm{E}-12$ & I.30E-II \\
\hline 18 & Thyroid hormone signaling pathway & $2.84 \mathrm{E}-12$ & 3.0IE-II \\
\hline 19 & VEGF signaling pathway & $3.7 \mid \mathrm{E}-12$ & $3.73 \mathrm{E}-\mathrm{II}$ \\
\hline 20 & HIF-I signaling pathway & $5.14 \mathrm{E}-12$ & $4.90 \mathrm{E}-\mathrm{II}$ \\
\hline 21 & Sphingolipid signaling pathway & $6.09 \mathrm{E}-12$ & $5.54 \mathrm{E}-\mathrm{II}$ \\
\hline 22 & Glioma & $8.26 \mathrm{E}-12$ & 7.I7E-II \\
\hline 23 & Pathways in cancer & I.0IE-II & $8.40 \mathrm{E}-1 \mathrm{I}$ \\
\hline 24 & Non-small cell lung cancer & $3.19 E-I 1$ & $2.54 \mathrm{E}-10$ \\
\hline 25 & Epstein-Barr virus infection & 4.87E-II & $3.72 \mathrm{E}-10$ \\
\hline 26 & Ras signaling pathway & $8.53 \mathrm{E}-\mathrm{II}$ & $6.26 \mathrm{E}-10$ \\
\hline 27 & cAMP signaling pathway & $9.40 \mathrm{E}-\mathrm{II}$ & $6.65 \mathrm{E}-10$ \\
\hline 28 & Colorectal cancer & $1.03 \mathrm{E}-10$ & $7.02 \mathrm{E}-10$ \\
\hline 29 & Chagas disease (American trypanosomiasis) & I.79E-10 & $1.18 \mathrm{E}-09$ \\
\hline 30 & TNF signaling pathway & $2.28 \mathrm{E}-10$ & I.45E-09 \\
\hline 31 & PI3K-Akt signaling pathway & $2.84 \mathrm{E}-10$ & I.75E-09 \\
\hline 32 & Endometrial cancer & $3.34 \mathrm{E}-10$ & I.99E-09 \\
\hline 33 & Cholinergic synapse & 4.IIE-IO & $2.38 \mathrm{E}-09$ \\
\hline 34 & HTLV-I infection & $6.66 \mathrm{E}-10$ & $3.74 \mathrm{E}-09$ \\
\hline 35 & Viral carcinogenesis & I.49E-09 & 8.13E-09 \\
\hline 36 & Fc gamma R-mediated phagocytosis & $3.05 \mathrm{E}-09$ & I.62E-08 \\
\hline
\end{tabular}


Table Continued

\begin{tabular}{|c|c|c|c|}
\hline S. No. & Pathway & P Value & Benjamini \\
\hline 37 & Renal cell carcinoma & $3.33 \mathrm{E}-09$ & I.72E-08 \\
\hline 38 & Hepatitis C & $3.97 \mathrm{E}-09$ & $2.00 \mathrm{E}-08$ \\
\hline 39 & Insulin signaling pathway & $6.27 \mathrm{E}-09$ & $3.07 \mathrm{E}-08$ \\
\hline 40 & cGMP-PKG signaling pathway & 6.57E-09 & $3.14 \mathrm{E}-08$ \\
\hline 41 & Chronic myeloid leukemia & $9.34 \mathrm{E}-09$ & $4.35 \mathrm{E}-08$ \\
\hline 42 & Influenza A & I.2IE-08 & $5.50 \mathrm{E}-08$ \\
\hline 43 & Acute myeloid leukemia & I.44E-08 & $6.39 \mathrm{E}-08$ \\
\hline 44 & Chemokine signaling pathway & $2.85 \mathrm{E}-08$ & I.24E-07 \\
\hline 45 & Toll-like receptor signaling pathway & $3.70 \mathrm{E}-08$ & I.57E-07 \\
\hline 46 & Long-term potentiation & $6.38 \mathrm{E}-08$ & $2.65 \mathrm{E}-07$ \\
\hline 47 & Tuberculosis & I.26E-07 & 5.IIE-07 \\
\hline 48 & Melanogenesis & $2.3 \mid \mathrm{E}-07$ & $9.18 \mathrm{E}-07$ \\
\hline 49 & Dopaminergic synapse & $2.63 \mathrm{E}-07$ & $1.02 \mathrm{E}-06$ \\
\hline 50 & FoxO signaling pathway & 4.19E-07 & $1.60 \mathrm{E}-06$ \\
\hline 51 & Insulin resistance & $4.78 \mathrm{E}-07$ & I.79E-06 \\
\hline 52 & Pancreatic cancer & $8.12 \mathrm{E}-07$ & $2.98 \mathrm{E}-06$ \\
\hline 53 & RapI signaling pathway & $9.13 \mathrm{E}-07$ & $3.29 \mathrm{E}-06$ \\
\hline 54 & Amphetamine addiction & $9.15 \mathrm{E}-07$ & $3.24 \mathrm{E}-06$ \\
\hline 55 & Adrenergic signaling in cardiomyocytes & $9.94 \mathrm{E}-07$ & $3.45 \mathrm{E}-06$ \\
\hline 56 & Inflammatory mediator regulation of TRP channels & $2.00 \mathrm{E}-06$ & $6.81 \mathrm{E}-06$ \\
\hline 57 & Wnt signaling pathway & $4.58 \mathrm{E}-06$ & I.53E-05 \\
\hline 58 & mTOR signaling pathway & $4.72 \mathrm{E}-06$ & I.55E-05 \\
\hline 59 & Alcoholism & $6.59 \mathrm{E}-06$ & $2.13 \mathrm{E}-05$ \\
\hline 60 & Calcium signaling pathway & $7.34 \mathrm{E}-06$ & $2.34 \mathrm{E}-05$ \\
\hline 61 & Gap junction & 8.29E-06 & $2.60 \mathrm{E}-05$ \\
\hline 62 & Platelet activation & $2.06 \mathrm{E}-05$ & $6.33 \mathrm{E}-05$ \\
\hline 63 & Signaling pathways regulating pluripotency of stem cells & 3.7IE-05 & I.I3E-04 \\
\hline 64 & Non-alcoholic fatty liver disease (NAFLD) & $6.73 \mathrm{E}-05$ & $2.01 \mathrm{E}-04$ \\
\hline 65 & Progesterone-mediated oocyte maturation & $6.96 \mathrm{E}-05$ & $2.04 \mathrm{E}-04$ \\
\hline 66 & NF-kappa B signaling pathway & $6.96 \mathrm{E}-05$ & $2.04 \mathrm{E}-04$ \\
\hline 67 & Toxoplasmosis & $7.08 \mathrm{E}-05$ & 2.05E-04 \\
\hline 68 & Circadian entrainment & I.22E-04 & $3.48 \mathrm{E}-04$ \\
\hline 69 & Epithelial cell signaling in Helicobacter pylori infection & I.30E-04 & 3.65E-04 \\
\hline 70 & Glucagon signaling pathway & $1.58 \mathrm{E}-04$ & $4.38 \mathrm{E}-04$ \\
\hline 71 & Adherens junction & $\mathrm{I} .80 \mathrm{E}-04$ & $4.90 \mathrm{E}-04$ \\
\hline 72 & Melanoma & I.80E-04 & $4.90 \mathrm{E}-04$ \\
\hline 73 & Bacterial invasion of epithelial cells & $3.02 \mathrm{E}-04$ & $8.12 \mathrm{E}-04$ \\
\hline 74 & Salmonella infection & $4.23 \mathrm{E}-04$ & 0.001122189 \\
\hline 75 & Leukocyte transendothelial migration & $4.68 \mathrm{E}-04$ & 0.00122285 \\
\hline 76 & MicroRNAs in cancer & $4.98 \mathrm{E}-04$ & 0.001284737 \\
\hline 77 & Long-term depression & $6.82 \mathrm{E}-04$ & 0.001735164 \\
\hline 78 & Apoptosis & $7.93 \mathrm{E}-04$ & 0.001991496 \\
\hline 79 & Regulation of actin cytoskeleton & $8.25 \mathrm{E}-04$ & 0.002044609 \\
\hline
\end{tabular}

Citation: Anandaram H.A computational approach to identify microRNA (miRNA) based biomarker of Pharmacovariant from the regulation of disease pathology. MOJ Proteomics Bioinform. 2018;7(3):I52-170. DOI: 10.15406/mojpb.20।8.07.00227 
Table Continued

\begin{tabular}{|c|c|c|c|}
\hline S. No. & Pathway & P Value & Benjamini \\
\hline 80 & Central carbon metabolism in cancer & $9.17 \mathrm{E}-04$ & 0.002244285 \\
\hline 81 & Shigellosis & $9.17 \mathrm{E}-04$ & 0.002244285 \\
\hline 82 & Renin secretion & 9.17E-04 & 0.002244285 \\
\hline 83 & Measles & 9.57E-04 & 0.002311316 \\
\hline 84 & Aldosterone-regulated sodium reabsorption & 0.001105 & 0.002635727 \\
\hline 85 & Bladder cancer & 0.001336 & $0.003 \mid 47586$ \\
\hline 86 & Leishmaniasis & 0.001468 & 0.003416078 \\
\hline 87 & Amoebiasis & $0.00154 I$ & $0.00354|75|$ \\
\hline 88 & Pertussis & 0.001875 & 0.004259408 \\
\hline 89 & Serotonergic synapse & 0.001951 & 0.00437895 \\
\hline 90 & Glutamatergic synapse & 0.002234 & 0.004955541 \\
\hline 91 & Type II diabetes mellitus & $0.0024 I I$ & 0.005284985 \\
\hline 92 & Aldosterone synthesis and secretion & 0.002634 & 0.005708816 \\
\hline 93 & Amyotrophic lateral sclerosis (ALS) & 0.002803 & 0.006004984 \\
\hline 94 & AMPK signaling pathway & $0.003 \mid 42$ & 0.006655783 \\
\hline 95 & Small cell lung cancer & 0.00325 & 0.00681028 \\
\hline 96 & Dorso-ventral axis formation & 0.003837 & 0.007948938 \\
\hline 97 & NOD-like receptor signaling pathway & 0.003969 & 0.008133865 \\
\hline 98 & Regulation of lipolysis in adipocytes & 0.004236 & 0.008588374 \\
\hline 99 & Thyroid cancer & 0.004712 & 0.009451116 \\
\hline 100 & Herpes simplex infection & 0.005813 & 0.01153254 \\
\hline 101 & Prion diseases & 0.006801 & 0.013346725 \\
\hline 102 & Jak-STAT signaling pathway & 0.007286 & 0.01415115 \\
\hline 103 & Bile secretion & 0.008878 & 0.017057979 \\
\hline 104 & Oocyte meiosis & 0.009287 & 0.017662609 \\
\hline 105 & Adipocytokine signaling pathway & 0.009333 & 0.017575749 \\
\hline 106 & Vascular smooth muscle contraction & 0.013248 & 0.024664527 \\
\hline 107 & Alzheimer's disease & 0.014431 & 0.026595965 \\
\hline 108 & Endocrine and other factor-regulated calcium reabsorption & 0.015978 & 0.029148707 \\
\hline 109 & Axon guidance & 0.017142 & 0.030963584 \\
\hline 110 & TGF-beta signaling pathway & 0.017343 & 0.031033366 \\
\hline 111 & Insulin secretion & 0.018039 & 0.031972854 \\
\hline 112 & Cocaine addiction & 0.020058 & 0.035199369 \\
\hline 113 & Tight junction & 0.022995 & 0.039944759 \\
\hline 114 & Vibrio cholerae infection & 0.02466 & 0.042428996 \\
\hline 115 & Viral myocarditis & 0.029784 & 0.050698785 \\
\hline 116 & Retrograde endocannabinoid signaling & 0.031582 & 0.053257247 \\
\hline 117 & Inflammatory bowel disease (IBD) & 0.039998 & 0.066669451 \\
\hline 118 & PPAR signaling pathway & 0.044852 & 0.074003009 \\
\hline 119 & Transcriptional misregulation in cancer & 0.048809 & 0.079750661 \\
\hline 120 & Thyroid hormone synthesis & 0.049987 & 0.080967512 \\
\hline 121 & RIG-I-like receptor signaling pathway & 0.049987 & 0.080967512 \\
\hline 122 & Gastric acid secretion & 0.055396 & 0.08883728 \\
\hline
\end{tabular}


Table Continued

\begin{tabular}{|c|c|c|c|}
\hline S. No. & Pathway & PValue & Benjamini \\
\hline 123 & African trypanosomiasis & 0.057954 & 0.092112284 \\
\hline 124 & Antigen processing and presentation & 0.061074 & 0.096199891 \\
\hline 125 & Primary immunodeficiency & 0.061114 & 0.095498989 \\
\hline 126 & Huntington's disease & 0.077264 & 0.119205309 \\
\hline 127 & Dilated cardiomyopathy & 0.077477 & 0.118607609 \\
\hline 128 & Salivary secretion & 0.081853 & 0.124192439 \\
\hline 129 & Rheumatoid arthritis & 0.086334 & 0.129837543 \\
\hline 130 & Carbohydrate digestion and absorption & 0.088331 & 0.131776895 \\
\hline$|3|$ & $A B C$ transporters & 0.095613 & 0.141305009 \\
\hline 132 & Pancreatic secretion & 0.09798 & $0.143657|2|$ \\
\hline 133 & Basal transcription factors & 0.099317 & 0.144513494 \\
\hline
\end{tabular}

\section{Discussion}

Combinatorial Analysis of miRNA based pharmacovarient association in regulatory network of Psoriasis indicate the fact that has-miR-139-5p was involved in the $\mathrm{T}$ cell receptor signaling pathway and contribute to the disease pathology of Psoriasis. In this article the significance of hsa-miR-139-5p was analysed for the pharmacologically associated genes of psoriasis and in the mere future the significance of miRNA association with Pharmacology will be analysed with respect to the associated genes of other diseases.

\section{Materials and methods}

\section{PubMed}

PubMed is an online search engine with open access facility to refer MEDLINE for identifying references and abstracts on topics in biomedical and life sciences. The United States National Library of Medicine (NLM) at the National Institutes of Health maintains the database as part of the Entrez system to retrieve information. Most of the records in PubMed contain links to the complete article, in PubMed Central. ${ }^{13}$ Information regarding the indexed journals in MEDLINE can be found in the Catalog of NLM.

\section{Varse and pharmacomiR}

Pharmaco-miR Verified Sets (VerSe) databas ${ }^{14}$ is generated from manual data mining of original research articles including the search terms 'miRNA' and 'drug' in the PubMed database.

\section{Regnetwork}

RegNetwork ${ }^{15}$ is a data base that contains five types (Transcription Factor-Transcription factor, Transcription Factor-Gene, Transcription Factor-microRNA, microRNA-Transcription Factor) of transcriptional and posttranscriptional regulatory relationships for human and mouse.

\section{Cytoscape}

Cytoscape $\mathrm{e}^{16}$ software is used for network construction, visualization and analysis in bioinformatics with an open source platform for visualizing the interactions in molecular networks and integrating them with the profiles of gene expression. Additional features in cytoscape are available as plugins for network and molecular profiling.

\section{Cytohubba}

Cytohubba ${ }^{17}$ is a cytoscape plugin for performing the analyses of gene regulation \& protein-protein interaction involved in the process of cellular pathways in the process of signal transduction. Cytohubba ranks the nodes of network by topological methods like Radiality, Betweenness, Closeness, Bottleneck, EcCentricity and etc.

\section{miRmap}

miRmap ${ }^{18}$ software addresses the challenges in post transcriptional repression of miRNAs in human genome by evolutionary, probabilistic thermodynamic and sequence-based features.

\section{Triplex RNA}

Triplex $\mathrm{RNA}^{19}$ is a database of cooperating micrRNAs with their mutual targets. In this database miRNA target prediction is based on the analysis of predicted miRNA triplex with molecular dynamics simulations and differential modeling procedures in mathematics.

\section{DAVID}

The database for Annotation, Visualization and Integrated discovery (DAVID) contain complete information about functional annotation of genes. The current version of DAVID ${ }^{20,21}$ is 6.8 and it provides a set of comprehensive tools for functional annotation of genes.

\section{Methodology}

(miRNA based association with pharmaco-clinical variants):

a. Identify the disease associated genes from Varse.

b. Obtain the associated list of miRNAs and transcription factors for the disease associated genes from PharmacomiR \& Regnetworks.

c. Construct and analyze the network in Cytoscape.

d. Identify the miRNA based hub genes and transcription factors from cytohubba.

e. Identify the implication of miRNA in Regulatory network in miRmap \& miRNA Triplex.

f. Identify and analyze the gene associated pathways in DAVID.

\section{Acknowledgements}

None. 


\section{Conflict of interest}

Author declares there is no conflict of interest.

\section{References}

1. Yaqoob P. Fatty acids as gatekeepers of immune cell regulation. Trends Immunol. 2003;24(12):639-645.

2. Ortonne JP. Aetiology and pathogenesis of psoriasis. Br J Dermatol. 1996;135(Suppl 49):1-5.

3. Xia J, Joyce CE, Bowcock AM, et al. Mutation of the variant alphatubulin TUBA 8 results in polymicrogyria with optic nerve hypoplasia. Hum Mol Genet. 2013;22:737-744.

4. Berezikov E, Chung WJ, Wills J, et al. Mammalian mirtron genes. Mol Cell. 2007;28:328-336.

5. Castellano L, Stebbing J. Deep sequencing of small RNAs identifies canonical and non-canonical miRNA and endogenous siRNAs in mammalian somatic tissues. Nucleic Acids Res. 2013;41(5):3339-3351.

6. Xia J, Zhang W. Noncanonical MicroRNAs and Endogenous siRNAs in Lytic Infection of Murine Gammaherpesvirus. PLoS One 2012;7(10):e47863

7. Morin RD, Connor MDO, Griffith M, et al. Application of massively parallel sequencing to microRNA profiling and discovery in human embryonic stem cells. Genome Res. 2008;18(4):610-621.

8. Neilsen CT, Goodall GJ, Bracken CP. IsomiRs - the overlooked repertoire in the dynamic microRNAome. Trends Genet. 2012;28(11):544-549.

9. Landgraf P, Rusu M, Sheridan R, et al. A Mammalian microRNA Expression Atlas Based on Small RNA Library Sequencing. Cell. 2007;129(7):1401-1414

10. Zheng $\mathrm{H}, \mathrm{Fu} \mathrm{R}$, Wang JT, et al. Advances in the Techniques for the Prediction of microRNA Targets. Int J Mol Sci. 2013;14(4):8179-8187.

11. Ruby JG, Jan C, Player C, et al. Large-Scale Sequencing Reveals 21U-RNAs and Additional MicroRNAs and Endogenous siRNAs in $C$. elegans. Cell. 2006;127(6):1193-1207.

12. Johansen C, Rittig AH, Mose M, et al. STAT2 is involved in the pathogenesis of psoriasis by promoting CXCL11 and CCL5 production by keratinocytes. PLoS One. 2017;12(5):e0176994.

13. Roberts RJ. PubMed Central: The GenBank of the published literature. Proc Natl Acad Sci U S A. 2001;98(2):381-382.

14. Piñero J, Bravo À, Queralt-Rosinach N, et al. DisGeNET: a discovery platform for the dynamical exploration of human diseases and their genes. Database (Oxford). 2015;2015:bav028

15. Amberger JS, Bocchini CA, Schiettecattle F, Scott AF, Hamosh A. OMIM.org: Online Mendelian Inheritance in $\operatorname{Man}\left(\mathrm{OMIM}^{\circledR}\right)$, an online catalog of human genes and genetic disorders. Nucleic Acids Res. 2015;43(Database issue):D789-D798

16. Liu ZP, Wu C, Miao H, et al. RegNetwork: an integrated database of transcriptional and post-transcriptional regulatory networks in human and mouse. Database (Oxford). 2015; bav095

17. Shannon P, Markiel A, Ozier O, et al. Cytoscape: a software environment for integrated models of biomolecular interaction networks. Genome Res. 2003;13(11):2498-2504.

18. Chin $\mathrm{CH}$, Chen $\mathrm{SH}, \mathrm{Wu} \mathrm{HH}$, et al. CytoHubba: identifying hub objects and subnetworks from complex interactome. BMC Syst Biol. 2014;8(Suppl. 4):S11.

19. Vejnar CE, Zdobnov EM. miRmap: Comprehensive prediction of microRNA target repression strength. Nucleic Acids Res. 2012;40(22):11673-11683.

20. Schmitz U, Lai $\mathrm{X}$, Winter $\mathrm{F}$, et al. Cooperative gene regulation by microRNA pairs and their identification using a computational workflow. Nucleic Acids Res. 2014;42(12):7539-7552.

21. Huang da W, Sherman BT, Lempicki RA. Systematic and integrative analysis of large gene lists using DAVID bioinformatics resources. Nat Protoc. 2009;4(1):44-57. 